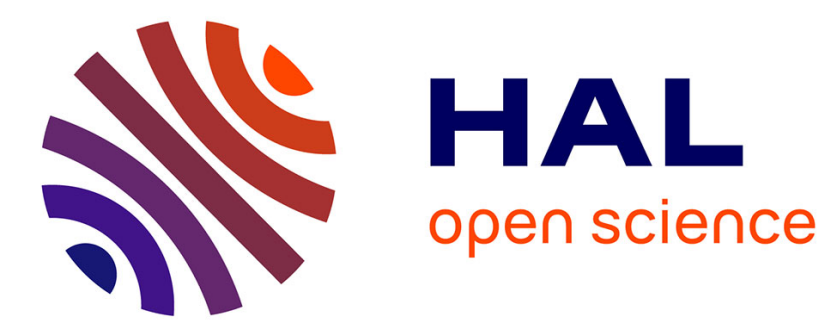

\title{
Sevrage tabagique en prévention secondaire d'un syndrome coronarien aigu: la place de la cigarette électronique
}

Yann Bellenguez

\section{- To cite this version:}

Yann Bellenguez. Sevrage tabagique en prévention secondaire d'un syndrome coronarien aigu: la place de la cigarette électronique. Sciences du Vivant [q-bio]. 2017. dumas-01871751

\section{HAL Id: dumas-01871751 https://dumas.ccsd.cnrs.fr/dumas-01871751}

Submitted on 11 Sep 2018

HAL is a multi-disciplinary open access archive for the deposit and dissemination of scientific research documents, whether they are published or not. The documents may come from teaching and research institutions in France or abroad, or from public or private research centers.
L'archive ouverte pluridisciplinaire $\mathbf{H A L}$, est destinée au dépôt et à la diffusion de documents scientifiques de niveau recherche, publiés ou non, émanant des établissements d'enseignement et de recherche français ou étrangers, des laboratoires publics ou privés. 
UNIVERSITE D'AMIENS

UFR DE MEDECINE

Année 2017

Thèse $\mathrm{N}^{\circ} 2017$ - 99

THESE

POUR LE DIPLOME D'ETAT DE

DOCTEUR EN MEDECINE

Discipline : Médecine Générale

Présentée et soutenue publiquement le 27 septembre 2017 par

BELLENGUEZ Yann

Né le 8 juin 1989

SEVRAGE TABAGIQUE EN PREVENTION SECONDAIRE D’UN SYNDROME CORONARIEN AIGU :

LA PLACE DE LA CIGARETTE ELECTRONIQUE

JURY :

Président : $\quad$ Monsieur le Professeur Olivier GANRY

Juges : $\quad$ Madame le Professeur Claire ANDREJAK

Madame le Docteur Catherine SZYMANSKI

Monsieur le Docteur Jean SCHMIDT

Directeur : $\quad$ Monsieur le Docteur Florent CHEVALIER 


\section{$\underline{\text { Remerciements : }}$}

A Monsieur le Professeur Olivier GANRY

Professeur des Universités - Praticien Hospitalier

Epidémiologie, économie de la santé et Prévention

Responsable du service d'Epidémiologie, hygiène hospitalière et santé publique

Pôle «Biologie, pharmacie et santé des populations »

Vous m'avez fait l'honneur d'accepter la présidence de ma thèse, soyez assuré de ma profonde reconnaissance.

A Madame le Professeur Claire ANDREJAK

Professeur des Universités - Praticien Hospitalier

Pneumologie

Vous avez accepté de juger ma thèse, veuillez recevoir ma profonde gratitude.

A Madame le Docteur Catherine SZYMANSKI

Maître de Conférences des Universités - Praticien Hospitalier

Cardiologie

Vous avez accepté de juger ce travail, recevez mes sincères remerciements.

A Monsieur le Docteur Jean SCHMIDT

Maître de Conférences des Universités - Praticien Hospitalier

Médecine interne

Vous avez accepté de juger ce travail, veuillez agréer ma sincère reconnaissance.

A Monsieur le Docteur Florent CHEVALIER

Praticien Hospitalier

Cardiologie

Pour m'avoir accordé le privilège de diriger ma thèse, pour m'avoir suivi dans ce sujet qui me tenait à cœur, pour tes précieux conseils, pour ton implication et ton enseignement au cours de mon stage, un grand merci ! 
A Monsieur le Professeur Pierre DUHAUT

Vous m'avez reçu et accordé de votre temps pour l'élaboration des statistiques. Soyez assuré de mon plus grand respect et de ma reconnaissance.

A Amandine, merci pour ton soutien, ta disponibilité et ton aide précieuse à chaque étape de la rédaction de cette thèse, tes connaissances ont été un véritable atout. Mais surtout, merci de rendre ma vie si belle et notre avenir si réjouissant.

A mes parents, vous avez toujours cru en moi et vous m'avez tout donné pour me permettre d'être là aujourd'hui. Un immense merci !

A mon grand frère, avec qui j'ai tant partagé, merci de m'avoir enseigné le mental et le dépassement de soi.

Merci à chaque membre de ma famille et particulièrement à mes grands-mères qui sont toujours prêtes à faire plaisir.

A ma belle-famille, merci pour votre relecture attentive, la traduction, mais aussi pour votre accueil, votre générosité et surtout pour la bière, le fromage et le saucisson !

A Thomas et Cyril, mes amis de toujours, merci d'avoir égayé mes week-ends durant ces longues études, pour tous les moments passés ensemble, pour les dimanches matin... Dommage que vous supportiez l'OM... Et merci d'avoir pris un peu d'avance dans la perte de vos cheveux !

A tous mes amis rencontrés au cours de ces études, Lucie, Pierre, Audrey, Doudou, Charles, Fanny, Audrey, Lucie, Alex, Scru, Ivan et d'autres... Toutes ces années sont passées bien plus vite grâce à vous.

A toutes les équipes médicales, paramédicales et les patients rencontrés au cours de mes stages merci d'avoir contribué à ma formation. 


\section{Résumé :}

Introduction : Le sevrage tabagique est un enjeu majeur en prévention secondaire d'un syndrome coronarien aigu (SCA). La cigarette électronique (CE) a fait récemment son apparition parmi les moyens d'aide au sevrage. Nous avons étudié l'utilisation, l'efficacité et les souhaits de «médicalisation » de la CE dans ce contexte.

Méthodes : Etude épidémiologique prospective avec inclusion des patients adultes fumeurs hospitalisés du $1^{\text {er }}$ juin 2015 au 31 mars 2016 au Centre Hospitalier de Saint Quentin (02) pour un SCA (sauf angor instable), à l'exclusion des patients de plus de 75 ans et/ou avec antécédent personnel de SCA, questionnés à 1 an de leur SCA.

Résultats : 81 patients ont été inclus. 59 questionnaires (73\%) ont été renvoyés et analysés. L'âge moyen était de 53,3 ans. Il y avait 45 hommes (76,3\%) et 14 femmes (23,7\%). 11 patients $(18,6 \%)$ avaient utilisé au moins une fois la CE avant le SCA et également 11 $(18,6 \%)$ après. C'était le deuxième moyen de sevrage le plus utilisé après les patchs de nicotine. A un an du SCA, 39 patients (66,1\%) étaient sevrés : 63,6\% (7/11) des utilisateurs de CE après le SCA contre 66,7\% (32/48) des non-utilisateurs $(p=0,848)$. Après analyse multivariée, seul l'arrêt sans aide était associé au sevrage $(p=0,013)$. Les utilisateurs de CE étaient significativement plus jeunes, fumaient plus avant le SCA et avaient débuté le tabac plus tôt. 50\% des utilisateurs de CE auraient souhaité être informés par leur médecin traitant et $53,3 \%$ qu'elle soit vendue en pharmacie.

Conclusion : La CE est le deuxième moyen de sevrage le plus utilisé après un SCA. Des études de qualité sont indispensables pour mieux connaître son efficacité et sa sécurité. 


\section{Abstract:}

Introduction: Smoking cessation is a major issue in secondary prevention of acute coronary syndrome (ACS). The electronic cigarette (EC) has recently come up as a means to help with ceasing. We have studied the use, efficiency and wishes for a "medicalization" of the EC in this context.

Methods: Prospective epidemiological study with inclusion of adult smoker patients hospitalized from 1 June 2015 to 31 March 2016 at the St Quentin Hospital (Picardy, France) for an ACS (except unstable angina), excluding patients over 75 years and/or with personal history of ACS, interviewed 1 year after their ACS.

Results: 81 patients were included. 59 questionnaires (73\%) were returned and analyzed. The average age was 53.3 years. There were 45 men (76.3\%) and 14 women (23.7\%). 11 patients (18.6\%) had used the EC at least once before their ACS and $11(18.6 \%)$ had used it after. That was the second most used smoking cessation method after the nicotine patches. One year after their SCA, 39 patients (66.1\%) had stopped smoking: 63.6\% (7/11) of EC users after the ACS against $66.7 \%(32 / 48)$ of non-users $(p=0.848)$. After multivariate analysis, only cessation without help was associated with ceasing $(p=0.013)$. CE users were significantly younger, were smoking more before their ACS, and had started smoking earlier. 50\% of CE users would have appreciated to be better informed by their general practitioner and $53.3 \%$ expressed themselves in favor of a sale in pharmacies.

Conclusion: EC is the second most common means of smoking cessation after an ACS. Further comparative studies are essential to know more about its efficiency and safety. 


\section{Table des matières :}

Remerciements.............................................................4

Résumé..................................................................6

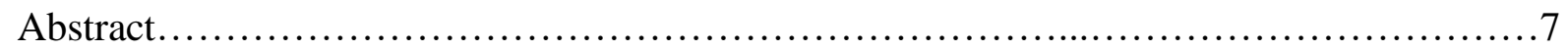

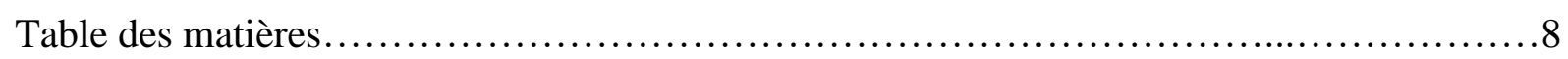

Lexique des abréviations....................................................... 9

I/ Introduction............................................................ 10

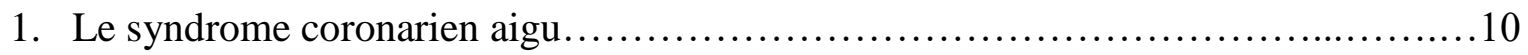

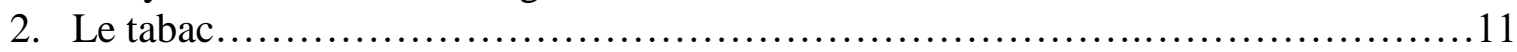

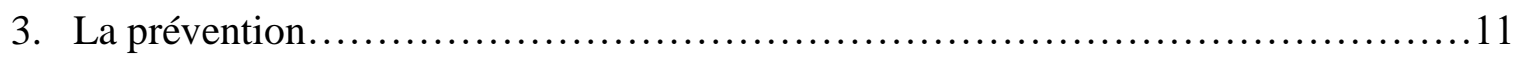

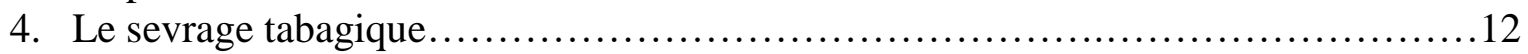

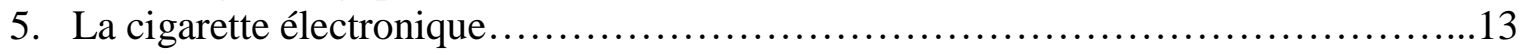

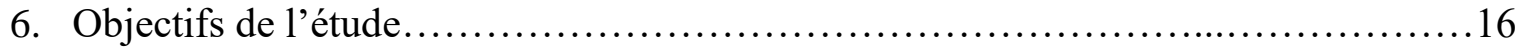

II/ Matériels et Méthodes......................................................... 17

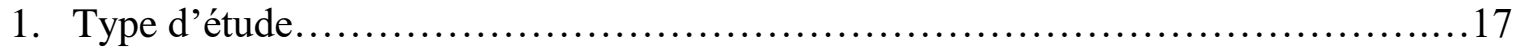

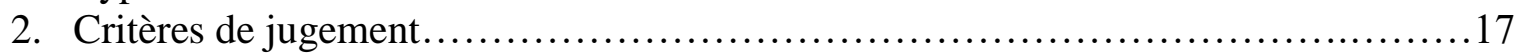

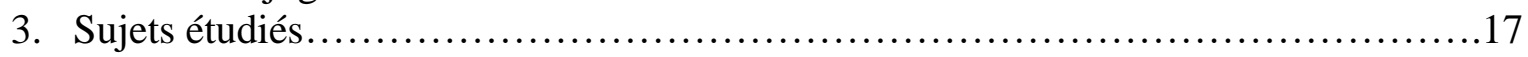

4. Recueil et gestion des données............................................. 18

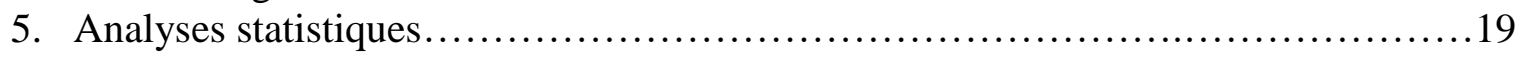

III/ Résultats.................................................................. 20

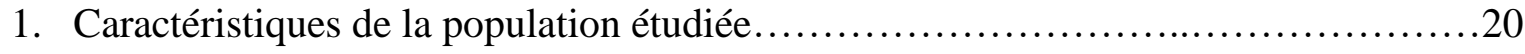

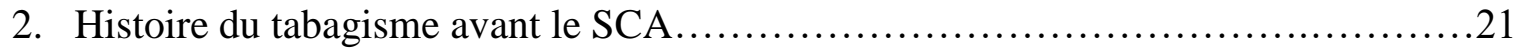

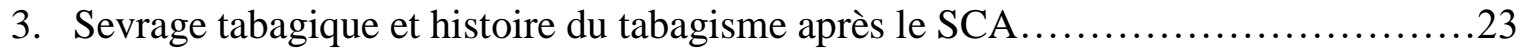

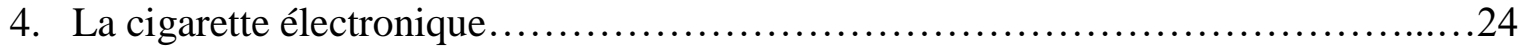

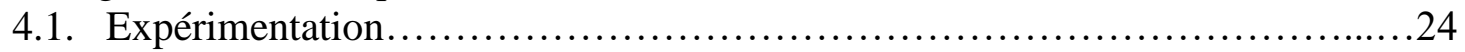

4.2. Caractéristiques des expérimentateurs.................................. 25

4.3. Efficacité et caractéristiques du sevrage tabagique chez les utilisateurs après le

SCA.....................................................................27

4.4. Perception du moyen le plus efficace....................................28

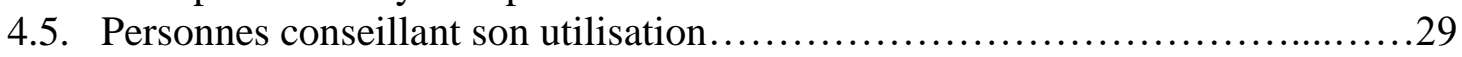

4.6. Rôle du système de santé souhaité par les patients......................... 30

4.7. Les non-utilisateurs de CE............................................ 31

IV/ Discussion............................................................ 33

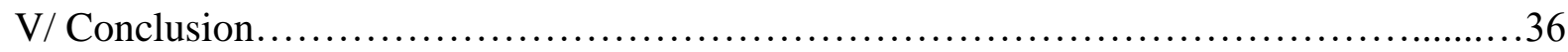

Références bibliographiques.................................................. 37

Annexe 1 : Aide à la cotation des actes du service de cardiologie du centre hospitalier de Saint Quentin..................................................................43

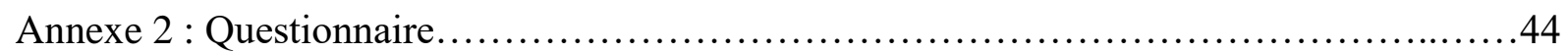

Annexe 3 : Test de Fagerström simplifié....................................... 51 


\section{Lexique des abréviations :}

AMM : Autorisation de mise sur le marché

AVC : Accident vasculaire cérébral

CE : Cigarette électronique

ECG : Electrocardiogramme

EI : Effets indésirables

HAS : Haute autorité de santé

HCSP : Haut conseil de la santé publique

HTA : Hypertension arterielle

INPES : Institut national de prévention et d'éducation pour la santé

OMS : Organisation mondiale de la santé

SCA : Syndrome coronarien aigu

SCA NST+ : Syndrome coronarien aigu sans sus-décalage du segment ST

SCA ST+ : Syndrome coronarien aigu avec sus-décalage persistant du segment ST

TCC : Thérapies cognitivo-comportementales

TNS : Traitements nicotiniques substitutifs 


\section{I/ Introduction}

\section{$\underline{1 .}$ Le syndrome coronarien aigu}

L'athérosclérose coronaire est une affection chronique inflammatoire marquée par l'apparition d'une plaque athéromateuse au sein des artères coronaires. L'érosion (ou la rupture) de cette plaque entraine l'activation de la coagulation, responsable d'un thrombus. Cette lésion peut entrainer une réduction brutale du flux sanguin coronaire d'aval : c'est le syndrome coronarien aigu (SCA). De nos jours, on classe les SCA en fonction de l'électrocardiogramme (ECG) initial en SCA avec sus-décalage persistant du segment ST (SCA ST+) (ce qui signifie que l'occlusion coronaire est totale) et en SCA sans sus-décalage du segment ST (SCA NST+) (quand la thrombose est sub-occlusive) qui regroupe l'angor instable et les infarctus sans onde Q [1]. On estime que chaque année en France, 100000 personnes sont atteintes d'un SCA [2-3]. Avec 32730 décès en 2014, la cardiopathie ischémique est une des premières causes de mortalité en France. Elle représente $6 \%$ de la mortalité totale [4].

L'amélioration de la prise en charge (appel au centre 15, transfert médicalisé par le SAMU, reperfusion précoce, antithrombotiques...) [5], a permis de réduire de $65 \%$ la mortalité précoce en 15 ans [6]. Cependant, le pronostic à moyen et long terme reste sombre : une étude de cohorte belge et britannique du registre GRACE a montré que, 5 ans après un SCA, la morbi-mortalité reste élevée : la mortalité toute cause était de $20 \%, 9 \%$ des patients ont présenté un nouveau SCA, 8\% un accident vasculaire cérébral (AVC), 17\% ont été réhospitalisés pour une revascularisation coronarienne et il y a eu en moyenne 1,6 réhospitalisations par patient [7].

La prise en charge au long cours, pouvant réduire cette morbi-mortalité, repose essentiellement sur la réadaptation cardiaque, le bon suivi du traitement médicamenteux «BASI» (bêtabloquant, antiagrégant plaquettaire, statine, inhibiteur de l'enzyme de conversion) et la lutte contre les facteurs de risque cardiovasculaires [8]. Parmi eux, le tabagisme, qui est retrouvé chez $40 \%$ des patients victimes d'un SCA. La Haute autorité de santé (HAS) définit comme un des objectifs prioritaires d'améliorer « le dépistage et débuter la prise en charge des patients tabagiques » [9]. 


\section{Le tabac}

Selon l'Organisation mondiale de la santé (OMS), actuellement, plus d'un milliard de personnes fument dans le monde [10]. Le tabac tue plus de 7 millions de personnes chaque année [11]. Un fumeur sur deux meurt prématurément des conséquences du tabagisme. Il est reconnu comme un facteur de risque de 6 des 8 premières causes de décès dans le monde. Il s'agit de la première cause de décès évitable [10]. En France, la mortalité annuelle imputable au tabagisme a été estimée à 78000 décès (59 000 hommes et 19000 femmes) en 2010 soit $14 \%$ de la mortalité totale. C'est une cause importante de décès prématurés puisque $27 \%$ des décès entre 35 et 69 ans ( 1 décès sur 3 chez les hommes et 1 sur 7 chez les femmes) est imputable au tabac [12]. Après une hausse constatée entre 2005 et 2010, la prévalence du tabagisme, en France, se stabilise en 2014 avec 34,1\% de fumeurs parmi les 15-75 ans. La proportion de fumeur quotidien concerne $28,2 \%$ de cette population soit $32,3 \%$ des hommes et $24,3 \%$ des femmes [13].

Le tabac constitue, avec l'hypertension artérielle (HTA), le diabète et les dyslipidémies, un des principaux facteurs de risque cardio-vasculaire [14]. Il est même souvent le seul facteur retrouvé dans les SCA du sujet jeune : $80 \%$ des victimes d'infarctus avant 45 ans sont des fumeurs et, entre 30 et 49 ans, le risque d'infarctus du myocarde chez le fumeur est multiplié par cinq [15]. Cependant, contrairement à l'âge, le sexe et les antécédents familiaux de maladies cardio-vasculaires, il s'agit d'un facteur de risque modifiable et donc accessible à des stratégies préventives.

\section{$\underline{\text { 3. La prévention }}$}

On distingue la prévention primaire, qui vise à empêcher l'apparition d'une maladie en luttant contre ses facteurs de risque et la prévention secondaire dont le but est de stopper, de retarder l'évolution d'une maladie ou de réduire le risque de rechute et de chronicité [16].

Devant la pandémie que constitue le tabagisme, des politiques de prévention sont instaurées à l'échelle mondiale avec le programme MPOWER de l'OMS, visant à surveiller la consommation de tabac et les politiques de prévention, à protéger la population contre la fumée du tabac, à offrir une aide à ceux qui veulent renoncer au tabac, à mettre en garde contre les dangers du tabagisme, à faire respecter l'interdiction de la publicité en faveur du tabac et à augmenter les taxes sur le tabac [10]. En France, un programme national de réduction du tabagisme a été édité par la ministre de la santé en septembre 2014 [17]. Il vient compléter de nombreuses mesures politiques ayant débuté avec la loi Veil de 1976 et de 
nombreuses campagnes d'information réalisées par l'Institut national de prévention et d'éducation pour la santé (INPES) ou des associations de lutte contre le tabac [13]. Les stratégies préventives ont démontré leur efficacité : une étude américaine conclut que la politique préventive de lutte contre le tabac, aux Etats-Unis, entre 1964 et 2012, a permis d'éviter 8 millions de décès prématurés et suggère que ces individus ont gagné 19,6 années de vie en moyenne [18] en limitant l'initiation et en encourageant le sevrage.

\section{$\underline{4 .}$ Le sevrage tabagique}

Les bénéfices de l'arrêt de l'exposition tabagique sont largement prouvés. Une étude menée chez les femmes montre, par exemple, que l'arrêt avant l'âge de 40 ans évite plus de 90\% de la surmortalité causée par la persistance du tabagisme. S'arrêter avant l'âge de 30 ans évite plus de $97 \%$ de celle-ci [19]. Concernant le SCA, le sevrage est un des principaux facteurs de réduction des décès [20-21] et des récidives : ce risque est divisé par 2 après un an d'abstinence et après 15 ans, le risque coronarien lié au tabac disparait [15]. Dans le cadre de la prévention secondaire, peu de démarches peuvent donner un bénéfice aussi rapide et important et cela pour un coût négligeable sans risque d'effet délétère. Pourtant, l'étude européenne EUROASPIRE IV souligne l'insuffisance de la prévention secondaire au moins 6 mois après un évènement coronarien avec, notamment près de la moitié des patients qui fumaient le mois précédant l'évènement qui poursuivent une intoxication tabagique et même $28 \%$ d'entre eux qui déclarent n'avoir pris aucune mesure pour tenter d'arrêter de fumer [22].

En effet, de nombreuses tentatives d'arrêt se concluent par une rechute. Parmi les fumeurs quotidiens de 15 à 75 ans en France, 70,7\% ont déjà fait une tentative d'arrêt du tabac volontaire pendant au moins 7 jours [13]. Après un an, seul 5\% des fumeurs ayant fait une tentative de sevrage, sans aide, y parviennent [23]. Les traitements nicotiniques substitutifs (TNS) augmentent les chances de sevrage à long terme de 50 à $70 \%$. Ces chances sont encore meilleures en cas d'association d'un patch avec une forme de délivrance rapide (gommes, pastilles, inhaleur ou spray nasal) [24]. Une des limites à l'ensemble de ces traitements est qu'ils ne traitent pas les aspects sensoriels et comportementaux du tabagisme manquant aux fumeurs lorsqu'ils cessent de fumer. 


\section{La cigarette électronique}

Dans le paysage des moyens de lutte contre le tabac, la cigarette électronique (CE), aussi appelée e-cigarette a fait récemment son apparition. Il s'agit d'un dispositif, breveté en 2003 par le pharmacien chinois Hon Lik [25], composé d'une batterie, un réservoir contenant le «e-liquide », un atomiseur pour convertir le liquide en aérosol et d'un embout buccal où le « vapoteur » viendra aspirer l'aérosol simulant la fumée. Ce dispositif vaporise, sans combustion, un aérosol contenant des taux variables de propylène glycol, glycérol, arômes, eau, alcool et nicotine [26]. Une très faible concentration plasmatique de nicotine était obtenue avec les $\mathrm{CE}$ de première génération (« cigalike »), qui sont très peu utilisées aujourd'hui. L'amélioration technologique a permis d'améliorer la biodisponibilité : des nicotinémies similaires voire supérieures aux cigarettes conventionnelles sont obtenues avec les CE de dernières générations [25].

Depuis 2009, le marché de la CE a connu un véritable essor en France [26]. Entre 2012 et 2014 : le nombre de boutiques spécialisées a été multiplié par 6 et le chiffre d'affaires par 3. Cependant, en 2015, on a constaté une baisse du dynamisme de ce marché [27]. En 2014, 6\% des français de 15 à 75 ans étaient vapoteurs (dont 2,9\% quotidiennement). Dans cette tranche d'âge, un quart des personnes l'ont déjà expérimenté et $23,4 \%$ de ceux qui l'ont essayé ont continué de l'utiliser régulièrement. La proportion d'expérimentateurs est décroissante de 25 à 75 ans [28].

Compte tenu de la diversité des produits disponibles sur le marché et de la modernisation rapide de ceux-ci, il est encore difficile d'évaluer avec certitude l'efficacité de la $\mathrm{CE}$ devant la difficulté de réaliser des études méthodologiquement acceptables. Les métaanalyses utilisant les premiers résultats d'essais comparatifs randomisés, basés cependant sur des dispositifs de CE dont la biodisponibilité de la nicotine est relativement faible, montrent, tout de même, que le taux d'abstinence à long terme est supérieur avec les CE contenant de la nicotine par rapport à celles n'en contenant pas ( $\mathrm{RR}=2,29$, IC 95\% : 1,05-4,96, $\mathrm{n}=662)$ [29]. La supériorité de la CE contenant de la nicotine versus patchs de nicotine n'est pas démontrée car statistiquement non significative ( $\mathrm{RR}=1,26$, IC $95 \%: 0,68$ à 2,34). Cependant la $\mathrm{CE}$ contenant de la nicotine apparait supérieure aux patchs pour réduire la consommation tabagique d'au moins $50 \%(\mathrm{RR}=1,41$, IC 95\% 1,20 à 1,67) [30]. Une autre méta-analyse retient également une supériorité de la CE avec nicotine versus sans nicotine dans le sevrage et la diminution de la consommation tabagique mais ne conclut pas sur cette supériorité versus thérapeutiques usuelles [31]. Enfin, une dernière méta-analyse remet en cause ces résultats, avec une probabilité plus faible d'atteindre le sevrage chez les utilisateurs de CE par 
rapport à ceux n'en utilisant pas $(\mathrm{OR}=0,72$, IC 95\%:0,57-0,91) [32] ; cette étude présente cependant plusieurs limites majeures à son interprétation : concernant la motivation des utilisateurs de la CE, l'analyse en sous-groupe concernant les fumeurs désireux d'arrêter de fumer montrait un résultat non significatif $(\mathrm{OR}=0,86$, IC 95\%:0,60-1,23); l'inclusion, dans l'analyse, d'un grand nombre d'études observationnelles avec un faible niveau de preuve, souvent sans groupe témoin; les études de bonnes qualités méthodologiques étant encore limitées [33].

En France, $82 \%$ des vapoteurs déclarent que la $\mathrm{CE}$ leur a permis de réduire leur consommation de tabac et qu'elle pourrait aider à arrêter de fumer [28]. En Europe, 35,1\% des vapoteurs actuels déclarent avoir arrêté de fumer grâce à elle [34]. Elle semble également efficace en prévention des rechutes chez les anciens fumeurs utilisant quotidiennement la $\mathrm{CE}$ [35].

Concernant le profil de sécurité de la $\mathrm{CE}$ : la nicotine, bien qu'elle participe à l'efficacité de la CE, représente des risques bien connus pour la santé : un potentiel addictif élevé [36], une majoration de la fréquence cardiaque et de la pression artérielle, une inhibition du développement pulmonaire chez le fœtus... [37]. Pourtant, les données concernant les effets indésirables (EI) graves avec les traitements nicotiniques existants se veulent rassurantes : il n'a pas été noté d'excès d'événements cardiovasculaires parmi les patients utilisant des patchs, y compris chez les patients atteints de maladies cardiovasculaires. Il existe tout de même un excès de douleurs thoraciques et de palpitations avec les TNS versus placebo (OR 1,88 (IC 95\%: 1,37 à 2,57)) mais ces évènements restent rares (2,5\% dans les groupes TNS versus 1,4\% dans les groupes témoins) [24]. Il n'a, par ailleurs, pas été retrouvé d'EI graves avec les TNS chez les femmes enceintes et les nouveau-nés [38]. Dans certains arômes réputés attractifs pour les jeunes, contenus dans la $\mathrm{CE}$, il a été constaté des composants réputés pour être à l'origine de pathologies respiratoires, notamment la bronchiolite oblitérante sévère (poumon des travailleurs de pop-corn) [39]. Cependant, nous ne savons pas si les taux mesurés ont une pertinence clinique. Pour le moment, aucun EI grave imputable à la CE n'a été retrouvé dans la méta-analyse de Hartmann-Boyce et al. (2016). Les EI les plus fréquemment rencontrés étaient l'irritation locale de la gorge et de la bouche [29]. Une étude a montré que des constituants toxiques retrouvés dans la vapeur des $\mathrm{CE}$ le sont à des niveaux 9 à 450 fois plus faibles que dans la fumée des cigarettes conventionnelles et même souvent comparables avec les traces retrouvées dans la préparation pharmaceutique de l'inhaleur Nicorette ${ }^{\circledR}$ [40]. Une récente étude a même montré qu'après une utilisation à long terme (au moins 6 mois), le taux de constituants cancérogènes et 
toxiques retrouvés dans la salive et les urines est bien plus faible dans les groupes utilisant uniquement la $\mathrm{CE}$ ou les TNS par rapport aux groupes utilisant du tabac ou ayant une consommation mixte (tabac plus TNS ou CE). On ne retrouvait pas des niveaux plus élevés de constituants toxiques chez les utilisateurs de CE seule par rapport aux utilisateurs de TNS. Au contraire, sur certaines mesures, l'usage de la CE était associé à des niveaux inférieurs [41].

Considérant que les constituants nocifs de la fumée de cigarette sont absents de la vapeur des $\mathrm{CE}$ ou à des niveaux nettement inférieurs à $5 \%$ des doses du tabac, mais que certains arômes et autres constituants de la CE peuvent être nocifs à long terme, la Public Health England estime que la CE est environ 95\% plus sûre que le tabagisme [42]. La Norwegian Institute of Public Health se veut plus prudente sur le risque de toxicité, notamment à cause de la nicotine. Elle considère cependant que les concentrations des cancérogènes sont très faibles dans les aérosols des $\mathrm{CE}$ et estime donc que le risque de cancer semble négligeable. Elle invite, tout de même, à plus de recherches en condition d'utilisation réelle pour conclure définitivement [37].

La HAS estime, tout de même, que les données sont encore insuffisantes pour la recommander dans le sevrage tabagique [43] et recommande d'en informer les vapoteurs. Elle déclare cependant qu'elle est supposée moins dangereuse que le tabac. Ainsi, si un fumeur refuse les moyens de substitution nicotinique recommandés, la CE ne doit pas être déconseillée si elle s'inscrit dans une stratégie d'arrêt ou de réduction du tabac avec accompagnement psychologique [44]. Plus récemment, le Haut Conseil de la Santé Publique (HCSP) a actualisé son avis relatif aux bénéfices-risques de la CE. Il recommande d'informer, sans en faire la publicité, les professionnels de santé et les fumeurs que la CE est un outil d'aide à l'arrêt du tabac chez les patients désireux d'arrêter de fumer et qu'elle constitue un outil de réduction des risques du tabagisme en usage exclusif (cette réduction n'est pas confirmée chez les utilisateurs concomitants de tabac et CE). Il demande aux parties prenantes, notamment à l'industrie pharmaceutique, d'engager une réflexion sur la création d'une CE « médicalisée » [45]. En effet, la CE pourrait, en théorie, être un médicament mais aucune demande d'autorisation de mise sur le marché (AMM) n'a été faite à ce jour. Elle est donc, pas défaut, considérée comme un produit de consommation courante « connexe » du tabac. Elle échappe, donc, aux études réglementaires basées sur des preuves exigées pour les produits de santé, mais elle est soumise à une réglementation particulière : la vente aux mineurs, la publicité (en dehors de la presse professionnelle et des lieux de vente) et le vapotage dans certains lieux (établissements accueillant des mineurs, moyens de transport collectifs fermés, espaces clos collectifs de travail) sont interdits [45]. 


\section{$\underline{\text { 6. Objectifs de l'étude }}$}

On sait qu'un nombre important de patients poursuit une intoxication tabagique après la survenue d'un SCA malgré les risques encourus. La cigarette électronique a fait récemment son apparition et pourrait participer à la réduction des risques liés au tabagisme. Nous avons voulu évaluer la place de la CE parmi les outils d'aide au sevrage tabagique chez les patients fumeurs ayant présenté un SCA.

Les objectifs secondaires étaient d'en évaluer l'efficacité sur le sevrage tabagique et de connaitre les souhaits de «médicalisation » de ce dispositif chez ces patients. 


\section{$\underline{\text { II/ Matériels et Méthodes }}$}

\section{Type d'étude}

Pour effectuer ce travail, nous avons réalisé une étude épidémiologique prospective monocentrique.

\section{Critères de jugement}

- Critère de jugement principal

Nombre de patients ayant utilisé la cigarette électronique pour l'aide au sevrage tabagique durant l'année suivant l'évènement coronarien aigu.

- Critères de jugement secondaires

-nombre de patients sevrés du tabac

-types d'outils d'aide au sevrage utilisés

-efficacité de la cigarette électronique sur le sevrage tabagique et la diminution de consommation tabagique chez les patients non sevrés

-attente des patients vis-à-vis du système de santé concernant la $\mathrm{CE}$

\section{$\underline{\text { 3. Sujets étudiés }}$}

- Critères d'inclusion

-patient âgé de 18 à 75 ans

-hospitalisé au Centre Hospitalier de Saint Quentin (Aisne, France) et ayant présenté un syndrome coronarien aigu du 1er juin 2015 au 31 mars 2016

-fumeurs au moment de l'évènement

-non opposition du patient après une information claire et adaptée orale et/ou écrite

- Critères d'exclusion

-antécédent personnel d'évènement coronarien aigu antérieur à l'inclusion

-codage d'angor instable

-patient dont le statut tabagique n'était pas précisément décrit et n'ayant pu être joint au téléphone 


\section{$\underline{4 . \text { Recueil et gestion des données }}$}

Nous avons inclus les patients, dans un premier temps, sur la base du codage établi en fin d'hospitalisation (cf. Annexe 1). La survenue d'un SCA est un évènement fort pour motiver le sevrage tabagique : par souci d'homogénéisation de notre population, les patients ayant déjà présenté un évènement coronarien aigu étaient exclus afin de ne pas sélectionner des patients réfractaires au sevrage. Nous avons également exclu les patients de plus de 75 ans, les résultats sur la prévalence et l'intérêt du sevrage au-delà de cet âge étant peu nombreux. A partir du dossier médical de ces patients, nous avons vérifié leur statut tabagique au moment de l'évènement et les éventuels antécédents coronariens. A la lecture des dossiers des patients ayant reçu le codage correspondant à l'angor instable, le diagnostic final était parfois douteux. Nous avons donc inclus uniquement les patients ayant présenté un SCA ST+ ou un SCA NST+ avec élévation de la troponine. Nous avons ensuite récupéré leurs coordonnées téléphoniques et postales.

Les patients inclus étaient contactés, par téléphone, un an après leur hospitalisation pour SCA. Ce contact permettait de présenter les médecins réalisant l'étude, d'expliquer notre étude et ses objectifs, d'obtenir le consentement oral du patient pour l'envoi du questionnaire par voie postale ou sur une adresse électronique, selon la préférence du patient. Pour les patients illettrés, le paragraphe d'information et les questions étaient lus lors de l'entretien téléphonique et le questionnaire rempli directement par l'investigateur. Pour les patients n'ayant pu être contactés par téléphone, le questionnaire était envoyé à l'adresse postale indiquée dans le dossier.

Le questionnaire (cf. Annexe 2, créé à partir de Google Forms pour le format électronique), était introduit par un paragraphe d'information avec la présentation des médecins participant à l'étude, les objectifs de l'étude et les modalités de recueil et d'analyse des données personnelles. Il comportait quatre parties : la première concernait les renseignements généraux (âge, sexe, situation professionnelle, niveau d'études), la deuxième et la troisième renseignaient respectivement l'histoire du tabagisme avant et après le SCA et la quatrième partie portait sur la $\mathrm{CE}$. La dépendance nicotinique était évaluée à l'aide du test de Fagerstöm simplifié (cf. Annexe 3), dans lequel fumer une cigarette roulée ou un cigarillo correspondait à 2 cigarettes classiques pour le calcul du degré de dépendance; le degré de motivation pour arrêter de fumer et le degré de confiance à ne pas rechuter était évalué à l'aide d'une échelle numérique de 0 à 10 . Une tentative de sevrage tabagique était définie comme un arrêt du tabac pendant au moins 7 jours. 


\section{$\underline{\text { 5. Analyses statistiques }}$}

Les analyses statistiques ont été réalisées avec le logiciel SAS. Les variables qualitatives dichotomiques ont été analysées avec le test du Chi ${ }^{2}$ et le test exact de Fischer, les variables qualitatives ordinales avec le test de Wilcoxon et les variables quantitatives continues avec un test $\mathrm{z}$ de comparaison de moyenne lorsque leur distribution était gaussienne et avec le test de Wilcoxon lorsqu'elle ne l'était pas. Une analyse multivariée par régression logistique a été réalisée pour évaluer l'efficacité des différents moyens d'aide sur le sevrage tabagique. Le risque $\alpha$ était fixé à $5 \%$. 


\section{III/ Résultats}

84 patients répondaient aux critères d'inclusion. 1 patient était décédé durant son hospitalisation et 2 autres au cours de l'année. 63 questionnaires sur 81 (78\%) ont été retournés. 4 questionnaires étaient incomplètement retournés ou comportaient des réponses incohérentes. 59 questionnaires (73\%) ont donc été analysés. Pour 4 questionnaires, certaines questions de la quatrième partie n'étaient parfois pas renseignées mais le reste des données était analysable.

\section{Caractéristiques de la population étudiée (Tableau 1)}

La population étudiée était constituée de $76,3 \%$ d'hommes $(n=45)$ et $23,7 \%$ de femmes $(\mathrm{n}=14)$. L'âge moyen était de 53,3 ans (identique chez les hommes et les femmes). 52,5\% de la population était active. Aucune différence significative n'a été mise en évidence entre les hommes et les femmes.

\begin{tabular}{lcccc}
\hline & Total (n=59) & Hommes (n=45) & Femmes (n=14) & \\
\hline $\begin{array}{l}\text { Age } \\
\text { Moyenne } \pm \text { écart-type }\end{array}$ & $53,3 \pm 9,0$ & $53,3 \pm 9,4$ & $53,3 \pm 8,1$ & $\mathrm{p}=0,971$ \\
\hline $\begin{array}{l}\text { Situation professionnelle } \\
\text { N }(\%)\end{array}$ & & & & \\
$-\quad$ Actifs & $31(52,5)$ & $23(51,1)$ & $8(57,1)$ & $\mathrm{p}=0,930$ \\
$-\quad$ Retraités & $10(16,9)$ & $7(15,6)$ & $3(21,4)$ & $\mathrm{p}=0,688$ \\
$-\quad$ Chômage / Sans activité & $14(23,7)$ & $12(26,7)$ & $2(14,3)$ & $\mathrm{p}=0,482$ \\
$-\quad$ Invalidité & $4(6,8)$ & $3(6,7)$ & $1(7,1)$ & $\mathrm{p}=1,000$ \\
\hline Niveau d'études & & & & \\
N $(\%)$ & & & & \\
$-\quad$ Sans diplôme & $15(25,4)$ & $12(26,7)$ & $3(21,4)$ & $\mathrm{p}=0,480$ \\
$-\quad$ Collège / CAP / BEP & $28(47,5)$ & $22(48,9)$ & $6(42,9)$ & \\
$-\quad$ Lycée / Baccalauréat & $6(10,2)$ & $4(8,9)$ & $2(14,3)$ & \\
$-\quad \geq$ Bac + 2 & $10(16,9)$ & $7(15,6)$ & $3(21,4)$ & \\
\hline
\end{tabular}

Tableau 1 : caractéristiques de la population étudiée 


\section{Histoire du tabagisme avant le SCA (Tableau 2)}

L’âge médian de début du tabagisme était de 16 ans. 4 patients sur 5 fumaient principalement des cigarettes classiques ou des tubes, les autres fumaient des cigarettes roulées ou des cigarillos et aucun ne fumait un autre type de tabac. Plus de la moitié de la population fumait du tabac plus de 20 fois par jour et $91,5 \%$ avait une dépendance à la nicotine $(\mathrm{n}=54) .59,3 \%$ des patients avaient fait au moins une tentative de sevrage tabagique avant leur SCA $(n=35)$ et $44,1 \%$ en avaient fait plusieurs $(n=26)$. Il n'y avait pas de différence significative entre les hommes et les femmes sur ces critères.

\begin{tabular}{|c|c|c|c|c|c|}
\hline \multirow{2}{*}{\multicolumn{2}{|c|}{$\begin{array}{l}\text { Age de début du tabagisme } \\
\text { Médiane (étendue) }\end{array}$}} & \multirow{2}{*}{$\begin{array}{l}\text { Total }(\mathbf{n = 5 9 )} \\
16(12-45)\end{array}$} & \multirow{2}{*}{$\begin{array}{c}\text { Hommes }(\mathbf{n}=\mathbf{4 5}) \\
17(12-35)\end{array}$} & \multicolumn{2}{|l|}{ Femmes $(n=14)$} \\
\hline & & & & $16(13-45)$ & $\mathrm{p}=0,878$ \\
\hline \multicolumn{6}{|c|}{ Type de tabac principal consommé } \\
\hline $\mathrm{N}(\%$ & $\begin{array}{l}\text { Cigarettes en paquets / tubes } \\
\text { Cigarettes roulées ou Cigarillos }\end{array}$ & $\begin{array}{l}47(79,7) \\
12(20,3)\end{array}$ & $\begin{array}{l}34(75,6) \\
11(24,4)\end{array}$ & $\begin{array}{c}13(92,9) \\
1(7,1)\end{array}$ & $\mathrm{p}=0,260$ \\
\hline \multicolumn{6}{|c|}{$\begin{array}{l}\text { Consommation moyenne quotidienne } \\
\text { de tabac }\end{array}$} \\
\hline- & $\begin{array}{l}0 \text { à } 5 \\
6 \text { à } 10 \\
11 \text { à } 15 \\
16 \text { à } 20 \\
21 \text { à } 30 \\
>30\end{array}$ & $\begin{array}{c}1(1,7) \\
4(6,8) \\
11(18,6) \\
13(22,0) \\
16(27,1) \\
14(23,7)\end{array}$ & $\begin{array}{c}1(2,2) \\
4(8,9) \\
8(17,8) \\
9(20,0) \\
12(26,7) \\
11(24,4)\end{array}$ & $\begin{array}{c}0 \\
0 \\
3(21,4) \\
4(28,6) \\
4(28,6) \\
3(21,4)\end{array}$ & $\mathrm{p}=0,826$ \\
\hline \multicolumn{6}{|c|}{ Score de Fagerström simplifié } \\
\hline $\mathrm{N}(\%$ & $\begin{array}{l}0 \text { à } 1: \text { pas de dépendance } \\
2 \text { à } 3: \text { dépendance modérée } \\
4 \text { à } 6: \text { dépendance forte }\end{array}$ & $\begin{array}{c}5(8,5) \\
17(28,8) \\
37(62,7)\end{array}$ & $\begin{array}{l}5(11,1) \\
12(26,7) \\
28(62,2)\end{array}$ & $\begin{array}{c}0 \\
5(35,7) \\
9(64,3)\end{array}$ & $\mathrm{p}=0,699$ \\
\hline \multicolumn{6}{|c|}{ Motivation au sevrage } \\
\hline- & $\begin{array}{l}0: \text { aucune } \\
1 \text { à } 3: \text { faible } \\
4 \text { à } 6: \text { modérée } \\
7 \text { à } 10: \text { forte }\end{array}$ & $\begin{array}{c}19(32,2) \\
15(25,4) \\
21(35,6) \\
4(6,8)\end{array}$ & $\begin{array}{c}13(28,9) \\
10(22,2) \\
18(40,0) \\
4(8,9)\end{array}$ & $\begin{array}{c}6(42,9) \\
5(35,7) \\
3(21,4) \\
0\end{array}$ & $\mathrm{p}=0,094$ \\
\hline
\end{tabular}

Tableau 2 : Histoire du tabagisme avant le SCA 
Les patients ayant fait au moins une tentative de sevrage $(n=35)$ ont essayé, en majorité, de se sevrer sans aide au sevrage (Figure 1). Parmi les aides au sevrage tabagique, les TNS étaient les moyens les plus utilisés avec 54,3\% d'expérimentateurs chez les patients ayant fait au moins une tentative de sevrage $(n=19)$ : ils avaient tous essayé les patchs et 9 d'entre eux ont utilisé des autres TNS en association. 31,4\% des patients ayant fait au moins une tentative de sevrage ont utilisé la CE $(n=11): 28,6 \%$ pour la CE avec nicotine $(n=10)$ et $5,7 \%$ pour la CE sans nicotine $(n=2)$ (1 patient a utilisé les 2$)$. Aucun patient n'a effectué de psychothérapie de soutien et/ou cognitivo-comportementale (TCC) ni utilisé la plateforme tabac-info-service ou autre application. Personne n'a essayé l'hypnose ou un autre moyen non cité dans le questionnaire. Il n'y avait pas de différence significative d'utilisation des différentes aides entre les hommes et les femmes.

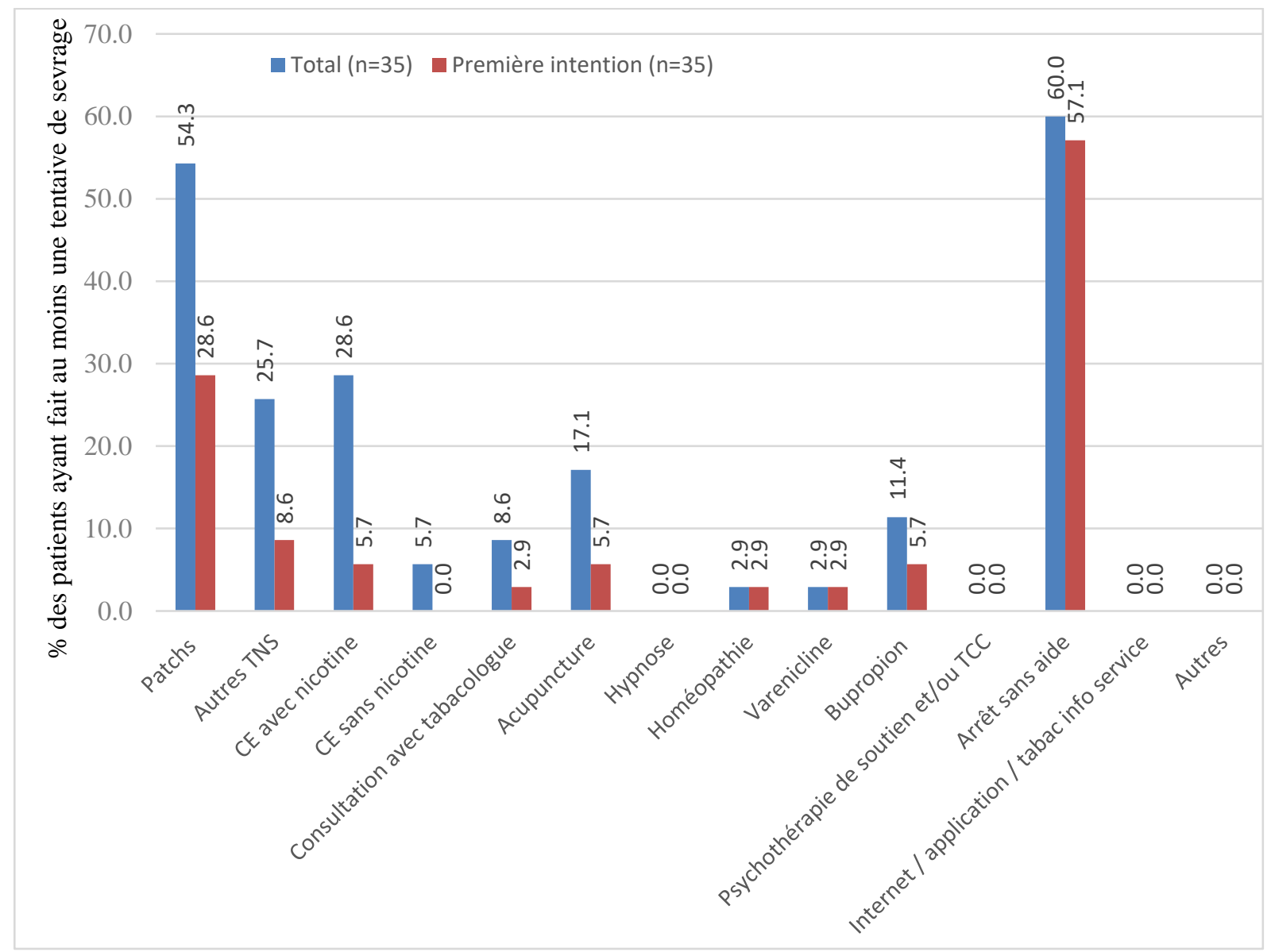

Figure 1 : Types d'aide au sevrage utilisés avant le SCA

\section{$\underline{\text { 3. Sevrage tabagique et histoire du tabagisme après le SCA }}$}

Après le SCA, 84,7\% des patients se disaient très motivés au sevrage $(n=50)(92,9 \%$ des femmes $(n=13)$ et $82,2 \%$ des hommes $(n=37))$. 1 an après le SCA, $66,1 \%$ des patients 
étaient sevrés $(\mathrm{n}=39)$ soit $62,2 \%$ des hommes $(\mathrm{n}=28)$ et 78,6\% des femmes $(\mathrm{n}=11)(p=0,342)$. Parmi eux, 92,3\% ont arrêté le tabac dès leur SCA $(n=36)$ et 94,9\% n'ont eu besoin que d'une tentative de sevrage $(n=37)$. Aucune différence significative n'a été mise en évidence entre les patients sevrés et non sevrés selon le sexe, l'âge, la situation professionnelle, le niveau d'étude, la consommation tabagique ou le score de Fagerström simplifié.

$40,7 \%$ des patients ont réussi à se sevrer sans aide au sevrage $(n=24)$, ils représentaient 61,5\% des patients sevrés. Les moyens d'aide les plus utilisés parmi tous les patients inclus étaient les TNS : 37,3\% des patients les ont utilisés $(n=22)$, ce qui représentait $62,9 \%$ des patients n'ayant pas réussi à se sevrer sans aide. 32,2\% ont utilisé des patchs $(n=19)$ et 15,3\% des autres TNS (n=9) (Figure 2). 18,6\% des patients ont utilisé la CE, soit 31,4\% des patients n'ayant pas réussi à se sevrer sans aide $(\mathrm{n}=11)$. 13,6\% ont utilisé la CE avec nicotine $(\mathrm{n}=8)$ et 6,8\% la CE sans nicotine ( $\mathrm{n}=4$ ) (1 patient a utilisé les 2). Peu de patients ont bénéficié d'une consultation avec un tabacologue $(6,8 \%(n=4))$ ou d'une psychothérapie $(1,7 \%(n=1))$. Aucun n'a utilisé de varenicline (Champix $\left.{ }^{\circledR}\right)$, de bupropion (Zyban $\left.{ }^{\circledR}\right)$ ni la plateforme tabac-infoservice ou autre application d'aide au sevrage. L'hypnose et l'acupuncture n'ont pas été utilisées. Aucune différence significative d'utilisation des différentes aides n'a été mise en évidence entre les 2 sexes.

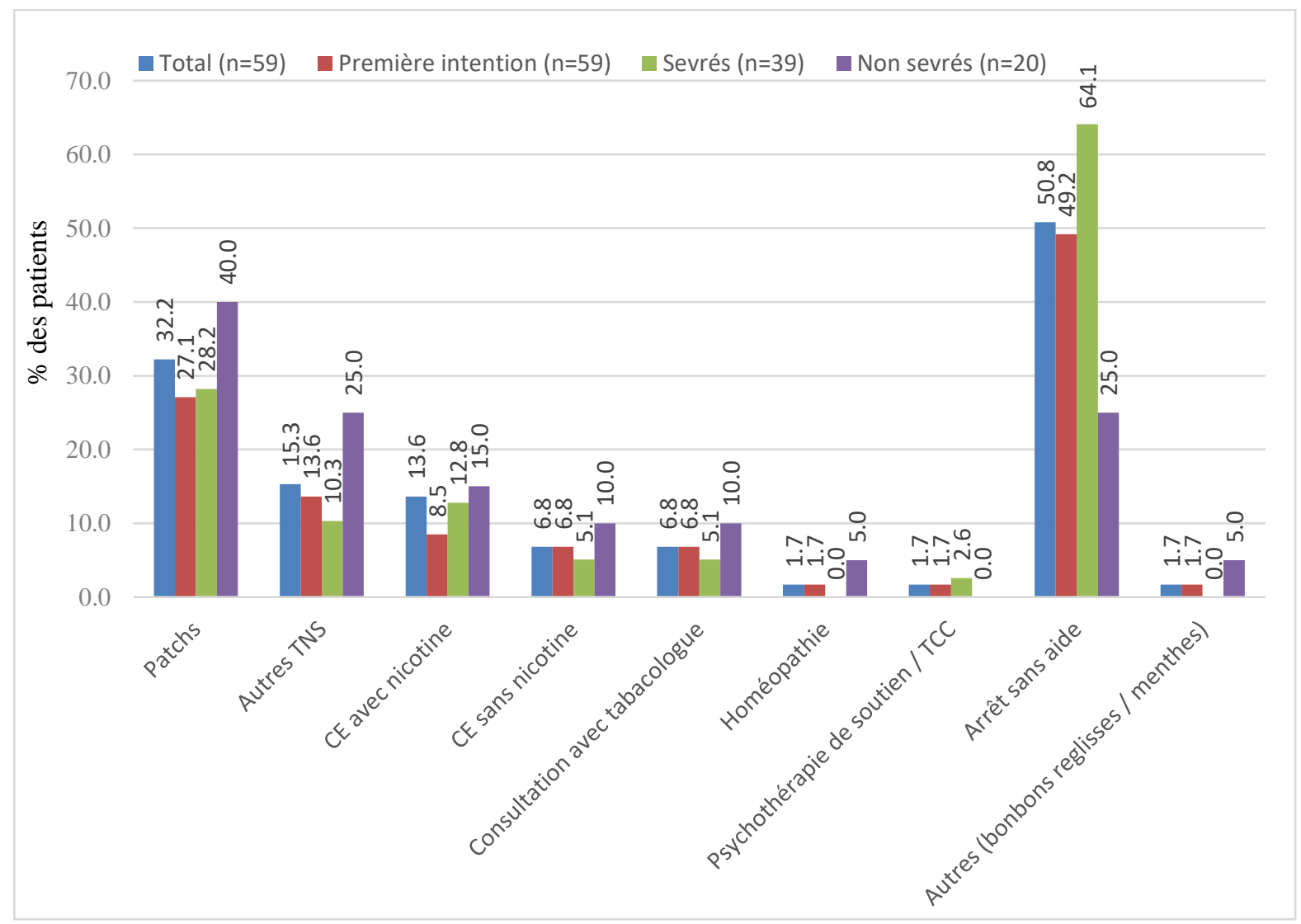

Figure 2 : Types d'aide au sevrage utilisés après le SCA 
Parmi les patients sevrés dès la première tentative $(n=37), 64,8 \%$ des patients n'ont pas utilisé d'aide au sevrage $(\mathrm{n}=24), 27 \%$ ont utilisé des TNS $(\mathrm{n}=10), 13,5 \%$ la CE $(\mathrm{n}=5)$ et $5,4 \%$ une consultation avec un tabacologue $(n=2)$. Les 2 patients ayant eu besoin d'une $2^{\text {e }}$ tentative ont utilisé à la fois des patchs et la CE pour se sevrer.

Parmi les patients non sevrés, 40\% n'ont pas essayé de moyen d'aide au sevrage $(n=8) .30 \%$ n'ont fait aucun arrêt du tabac pendant au moins 7 jours $(n=6)$ et $20 \%$ n'ont rien entrepris pour arrêter de fumer (aucune tentative d'arrêt et aucun moyen d'aide utilisé) (n=4). $85 \%$ ont diminué leur consommation tabagique $(n=17)$.

\section{La cigarette électronique}

\subsection{Expérimentation}

$28,8 \%$ des patients ont expérimenté la CE pour se sevrer $(n=17): 18,6 \%$ avant le SCA $(\mathrm{n}=11)$, soit $31,4 \%$ des patients ayant fait au moins une tentative de sevrage, et également 18,6\% après le SCA $(n=11) .5$ l'ont utilisée avant et après le SCA. 72,7\% des utilisateurs de $\mathrm{CE}$ avant le SCA ne l'ont pas utilisée en première intention mais après au moins un échec au sevrage $(n=8)$ ainsi que $27,3 \%$ des utilisateurs après le SCA $(n=3)$. Parmi eux, sur 16 réponses, 56,2\% l'utilisaient encore un an après le SCA (n=9) (Figure 3) soit 81,8\% des patients l'ayant utilisée pour le sevrage tabagique après le SCA. 25\% des utilisateurs l'ont arrêtée car elle ne les a pas suffisamment aidés $(\mathrm{n}=4)$, parmi eux $75 \%$ n'étaient pas sevrés à un an de leur SCA $(n=3)$.

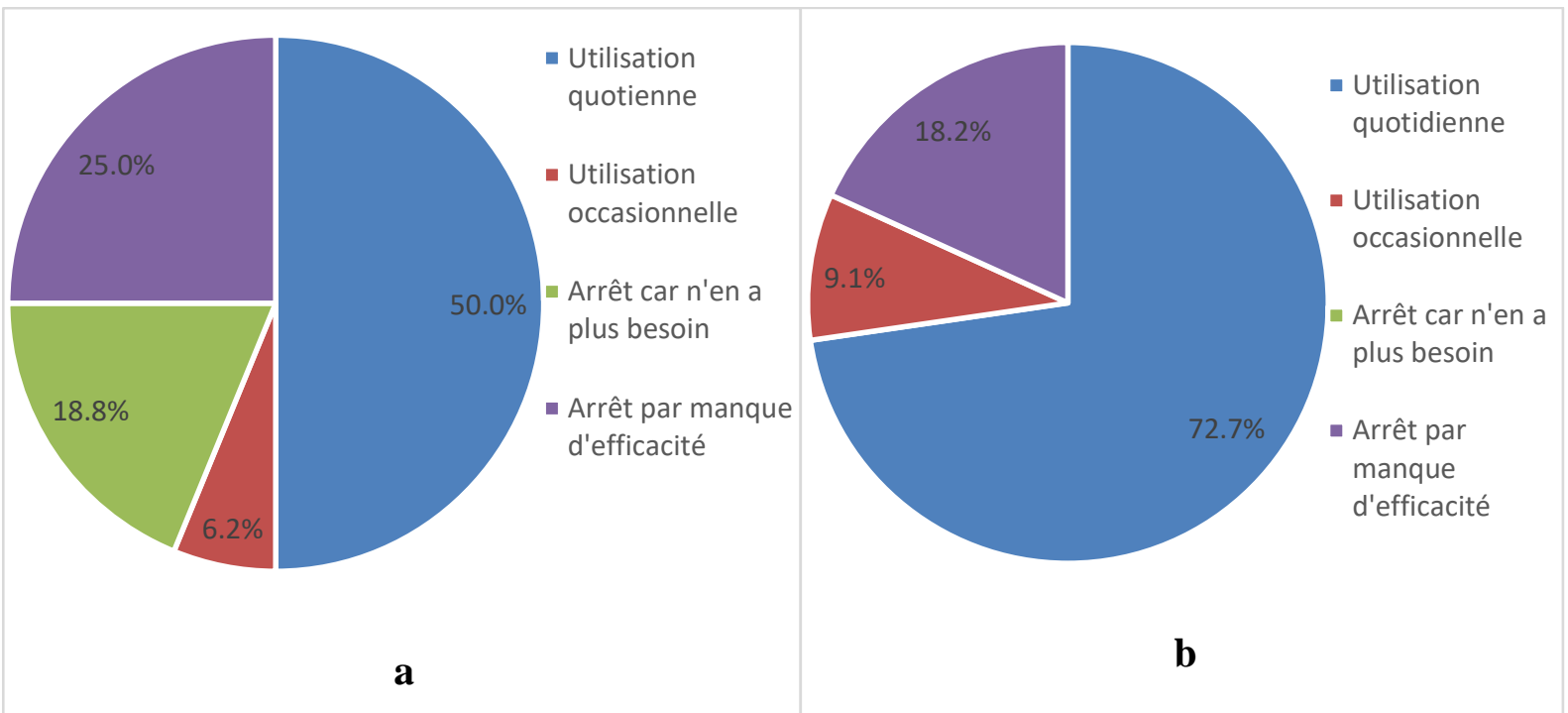

Figure 3 : persistance de l'utilisation de la $\mathrm{CE}$ :

a) Chez tous les expérimentateurs

b) Chez les utilisateurs de CE après le SCA 


\subsection{Caractéristiques des expérimentateurs}

$31,1 \%$ des hommes l'ont expérimentée $(\mathrm{n}=14)$ et $21,4 \%$ des femmes $(\mathrm{n}=3)(p=0,737)$. Les utilisateurs de CE, étaient significativement plus jeunes, avaient débuté le tabac plus tôt et fumaient plus de tabac avant le SCA que les non-utilisateurs (Tableau 3). 70,6\% des expérimentateurs de $\mathrm{CE}$ fumaient du tabac plus de 20 fois par jour et avaient une dépendance nicotinique forte $(n=12)$. Aucun retraité ne l'a essayé. 


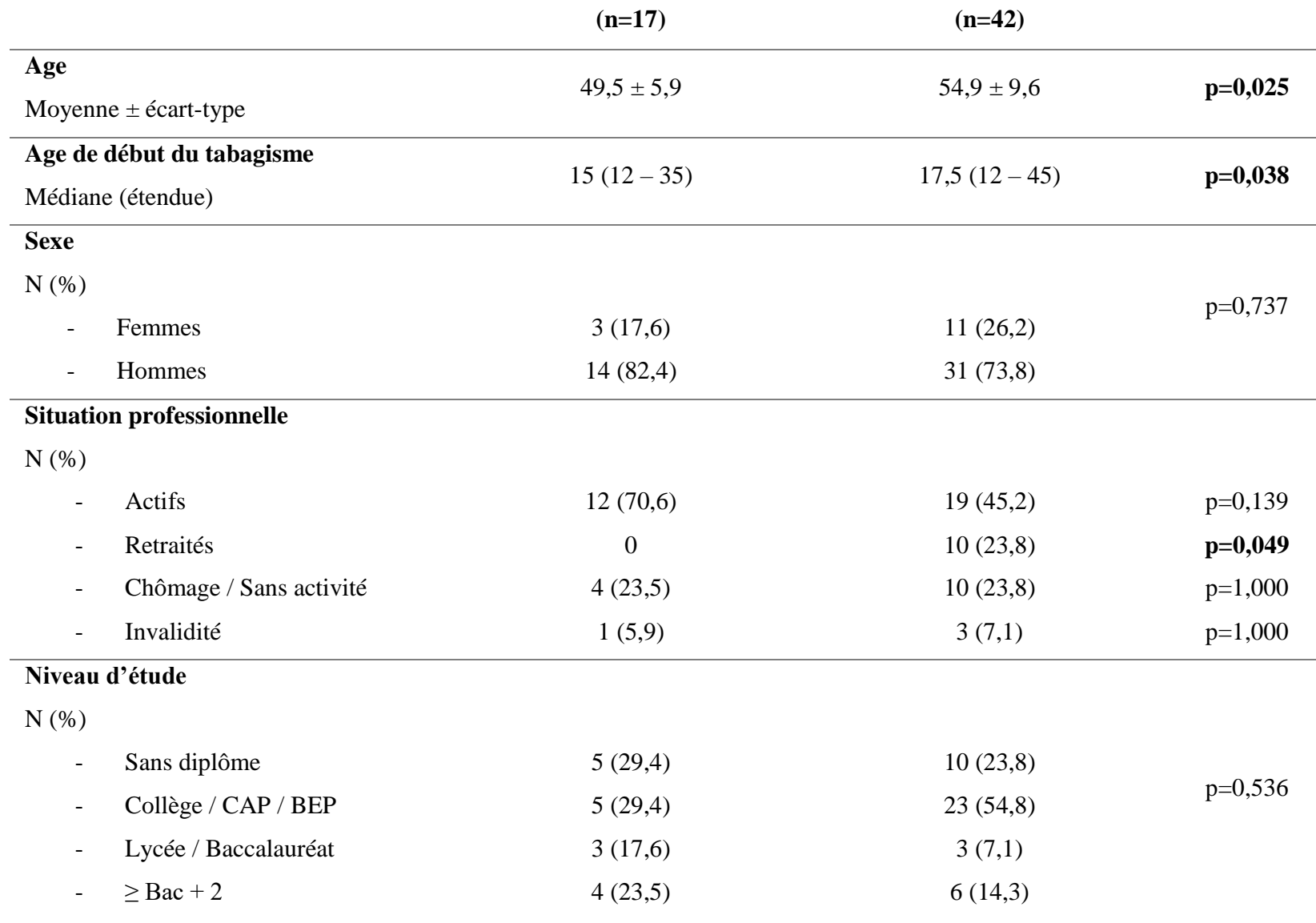

Consommation moyenne quotidienne

de tabac avant le SCA

$\mathrm{N}(\%)$

$\begin{array}{llccc}- & 0 \text { à } 5 & 0 & 1(2,4) & \\ - & 6 \text { à } 10 & 1(5,9) & 3(7,1) & \text { p=0,032 } \\ - & 11 \text { à } 15 & 0 & 11(26,2) & \\ - & 16 \text { à } 20 & 4(23,5) & 10(21,4) \\ - & 21 \text { à } 30 & 6(35,3) & 8(19,0) & \\ - & >30 & 6(35,3) & \end{array}$

Score de Fagerström simplifié

$\mathrm{N}(\%)$

\begin{tabular}{|c|c|c|c|}
\hline - $\quad 0-1:$ pas de dépendance & $1(5,9)$ & $4(9,5)$ & $\mathrm{p}=0,427$ \\
\hline 2-3: dépendance modérée & $4(23,5)$ & $13(31,0)$ & \\
\hline 4-6 : dépendance forte & $12(70,6)$ & $25(59,5)$ & \\
\hline
\end{tabular}

\section{Motivation à arrêter de fumer avant le}

SCA

$\mathrm{N}(\%)$

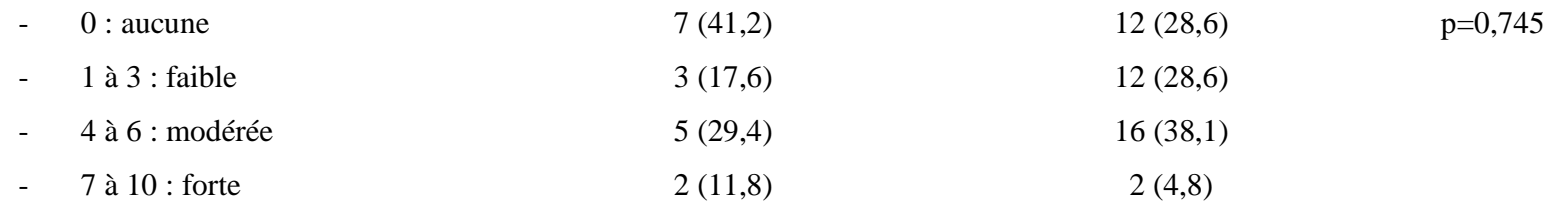

Tableau 3 : Caractéristiques des expérimentateurs de CE pour le sevrage versus non expérimentateurs. 
Après le SCA, 90,9\% des utilisateurs de CE se disaient très motivés au sevrage (Tableau 4). 63,6\% des patients ayant utilisé la CE comme moyen de sevrage étaient sevrés $(\mathrm{n}=7)$ contre $66,7 \%$ des non utilisateurs $(\mathrm{n}=32)(p=0,848)$ et contre $54,5 \%$ des patients ayant utilisé au moins un TNS $(\mathrm{n}=12)(p=0,719)$. Sur les 7 patients sevrés avec la CE, 42,9\% n'étaient pas sevrés immédiatement après le SCA $(n=3)$ et $28,6 \%$ ont essayé la $\mathrm{CE}$ après un premier échec au sevrage $(n=2)$. Les utilisateurs de CE ont tous fait au moins une tentative de sevrage $(n=11)$ et $27,3 \%$ en ont fait plusieurs $(n=3)$.

Les patients sevrés n'ayant pas utilisé la CE étaient tous sevrés depuis leur SCA $(\mathrm{n}=32) .87,5 \%$ des non-utilisateurs de $\mathrm{CE}$ ont fait au moins une tentative de sevrage après le SCA $(n=42)$ et $6,2 \%$ en ont fait plusieurs $(n=3)$. Les utilisateurs de CE ont ainsi fait significativement plus de tentatives de sevrage que les non-utilisateurs de $\mathrm{CE}(p=0,030)$.

Après analyse multivariée évaluant l'efficacité des différentes aides, seul l'arrêt sans aide était significativement associé au sevrage $(p=0,013)$.

\begin{tabular}{|c|c|c|c|c|}
\hline a) & & Utilisateurs de CE (n=11) & Non utilisateurs de $C E(n=48)$ & \\
\hline \multicolumn{5}{|c|}{$\begin{array}{l}\text { Motivation à arrêter de } \\
\text { fumer après le SCA }\end{array}$} \\
\hline \multicolumn{5}{|c|}{$\mathrm{N}(\%)$} \\
\hline- & $0:$ aucune & 0 & $1(2,1)$ & $\mathrm{p}=0,098$ \\
\hline- & 1 à 3 : faible & 0 & $1(2,1)$ & \\
\hline- & 4 à 6 : modérée & $1(9,1)$ & $6(12,5)$ & \\
\hline - & 7 à 10 : forte & $10(90,9)$ & $40(83,3)$ & \\
\hline
\end{tabular}

b)

Utilisateurs de CE sevrés Non utilisateurs de CE sevrés

$(\mathbf{n}=7) \quad(\mathrm{n}=32)$

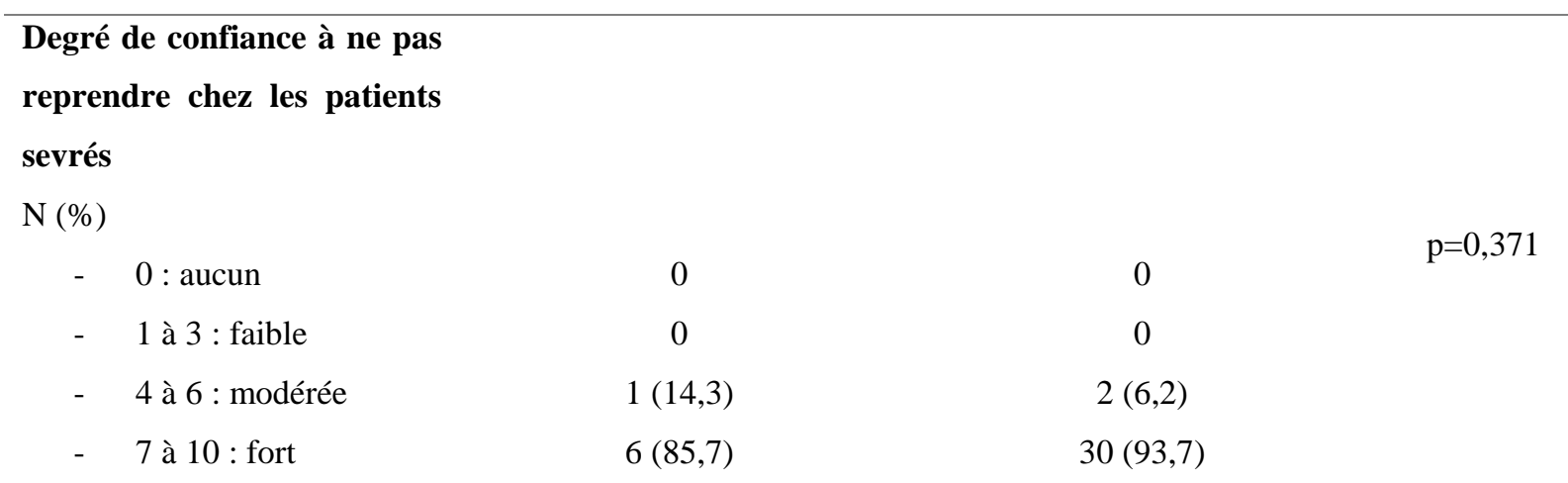

Tableau 4 : Motivation et confiance à l'arrêt du tabac :

a) chez les utilisateurs de $\mathrm{CE}$ et non utilisateurs après le SCA

b) chez les utilisateurs de CE sevrés et non utilisateurs sevrés après le SCA 
Parmi les patients non sevrés, 100\% des utilisateurs de CE $(n=4), 70 \%$ des utilisateurs de TNS $(\mathrm{n}=7)(p=0,505)$, et $81,2 \%$ des non-utilisateurs de CE $(\mathrm{n}=13)(p=1,000)$ ont réduit leur consommation tabagique.

\subsection{Perception du moyen le plus efficace}

40 patients ont répondu à cette question en citant parfois plusieurs moyens. De nombreux patients ont cité un moyen non thérapeutique, ainsi pour 47,5\% d'entre eux, la volonté et/ou la peur de la mort ou d'une récidive apparaissaient comme les moyens les plus efficaces $(\mathrm{n}=19)$ (Tableau 5). Les expérimentateurs de CE étaient 35,7\% à la considérer comme le moyen le plus efficace $(n=5)$ alors que les expérimentateurs de TNS étaient 34,8\% à les citer comme les plus efficaces $(\mathrm{n}=8)(p=1,000)$. Ceux qui ont essayé les 2 étaient 33,3\% à penser que la CE était le moyen le plus efficace $(n=3)$ contre $11,1 \%$ pour les TNS $(n=1)$.

\begin{tabular}{|c|c|c|c|c|c|c|}
\hline & $\begin{array}{l}\text { Total } \\
(n=40)\end{array}$ & $\begin{array}{l}\text { Sevrés } \\
(n=26)\end{array}$ & $\begin{array}{l}\text { Non sevrés } \\
\qquad(n=14)\end{array}$ & $\begin{array}{c}\text { Expérimentateurs } \\
\text { de CE }(n=14)\end{array}$ & $\begin{array}{c}\text { Expérimentateurs } \\
\text { de TNS }(n=23)\end{array}$ & $\begin{array}{c}\text { Expérimentateurs } \\
\text { de TNS et de CE } \\
(n=9)\end{array}$ \\
\hline TNS & $8(20)$ & $6(23,1)$ & $2(14,3)$ & $1(7,1)$ & $8(34,8)$ & $1(11,1)$ \\
\hline -Patchs & $7(17,5)$ & $5(19,3)$ & $2(14,3)$ & $1(7,1)$ & $7(30,4)$ & $1(11,1)$ \\
\hline - Autres TNS & $3(7,5)$ & $2(7,7)$ & $1(7,1)$ & 0 & $3(13,0)$ & 0 \\
\hline $\mathbf{C E}$ & $5(12,5)$ & $4(15,4)$ & $1(7,1)$ & $5(35,7)$ & $3(13)$ & $3(33,3)$ \\
\hline Bupropion & $1(2,5)$ & 0 & $1(7,1)$ & 0 & 0 & 0 \\
\hline $\begin{array}{l}\text { Consultation de } \\
\text { tabacologie }\end{array}$ & $1(2,5)$ & $1(3,8)$ & 0 & 0 & $1(4,3)$ & 0 \\
\hline Hypnose & $1(2,5)$ & 0 & $1(7,1)$ & 0 & 0 & 0 \\
\hline $\begin{array}{l}\text { Mental / volonté } \\
\text { / motivation }\end{array}$ & $15(37,5)$ & $11(42,3)$ & $4(28,6)$ & $4(28,6)$ & $6(26,1)$ & $2(22,2)$ \\
\hline $\begin{array}{l}\text { Peur (mort / } \\
\text { récidives) }\end{array}$ & $6(15)$ & $6(23,1)$ & 0 & $3(21,4)$ & $2(8,7)$ & 0 \\
\hline Au cas par cas & $1(2,5)$ & $1(3,8)$ & 0 & 0 & 0 & 0 \\
\hline Ne sait pas & $5(12,5)$ & $1(3,8)$ & $4(28,6)$ & $1(7,1)$ & $3(13,0)$ & $1(11,1)$ \\
\hline $\begin{array}{l}\text { Ne pas } \\
\text { commencer }\end{array}$ & $1(2,5)$ & 0 & $1(7,1)$ & $1(7,1)$ & $1(4,3)$ & $1(11,1)$ \\
\hline Aucun & $1(2,5)$ & 0 & $1(7,1)$ & $1(7,1)$ & $1(4,3)$ & $1(11,1)$ \\
\hline
\end{tabular}

Tableau 5 : Moyens d'aide au sevrage tabagique cités comme étant le plus efficace* *les données sont des effectifs (avec pourcentages) 


\section{$\underline{4.5 . P e r s o n n e s ~ c o n s e i l l a n t ~ s o n ~ u t i l i s a t i o n ~}$}

14 patients ont répondu à la question sur la personne ayant motivé l'utilisation de la CE. Elle a été, en majorité, motivée par la famille des patients $(57,1 \%(\mathrm{n}=8))$ (Figure 4). $35,7 \%$ des utilisateurs ont été motivés par un professionnel de santé et/ou leur médecin traitant $(n=5), 80 \%$ de ceux-ci ne sont pas sevrés $(n=4)$ et l'autre patient fumait plus de 20 fois par jour et avait une dépendance nicotinique forte avant le SCA. Certains patients ont cité plusieurs personnes.

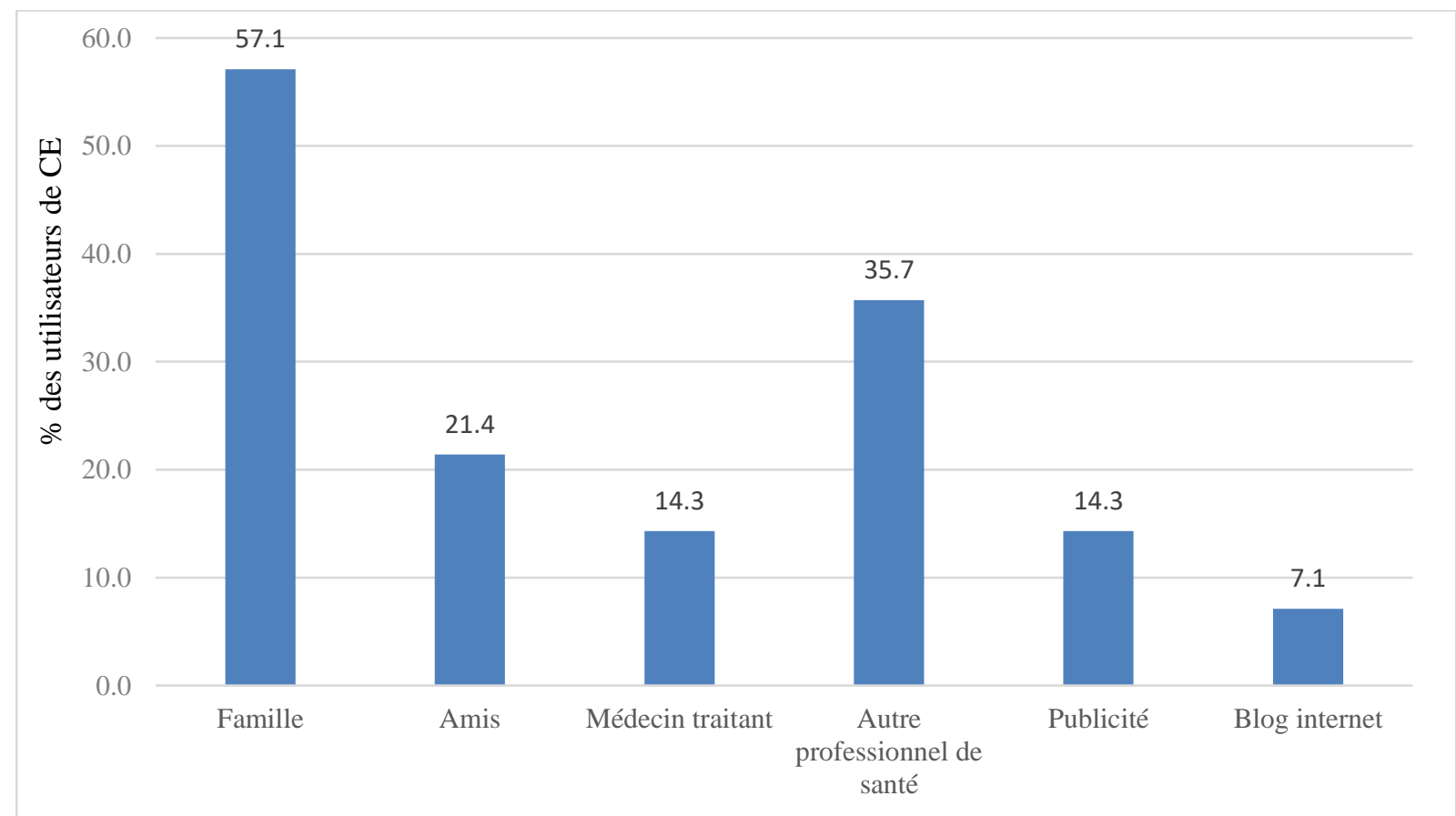

Figure 4 : personne(s) ayant motivé l'utilisation de la CE

16 patients ont répondu à la question sur la personne qui les a conseillés dans l'utilisation de la CE. Parmi eux, 25\% l'étaient par un professionnel de santé $(n=4)$ (3 d'entre eux avaient été motivés par un professionnel de santé). 31,2\% n'étaient conseillés par personne $(\mathrm{n}=5)$ (Figure 5). 


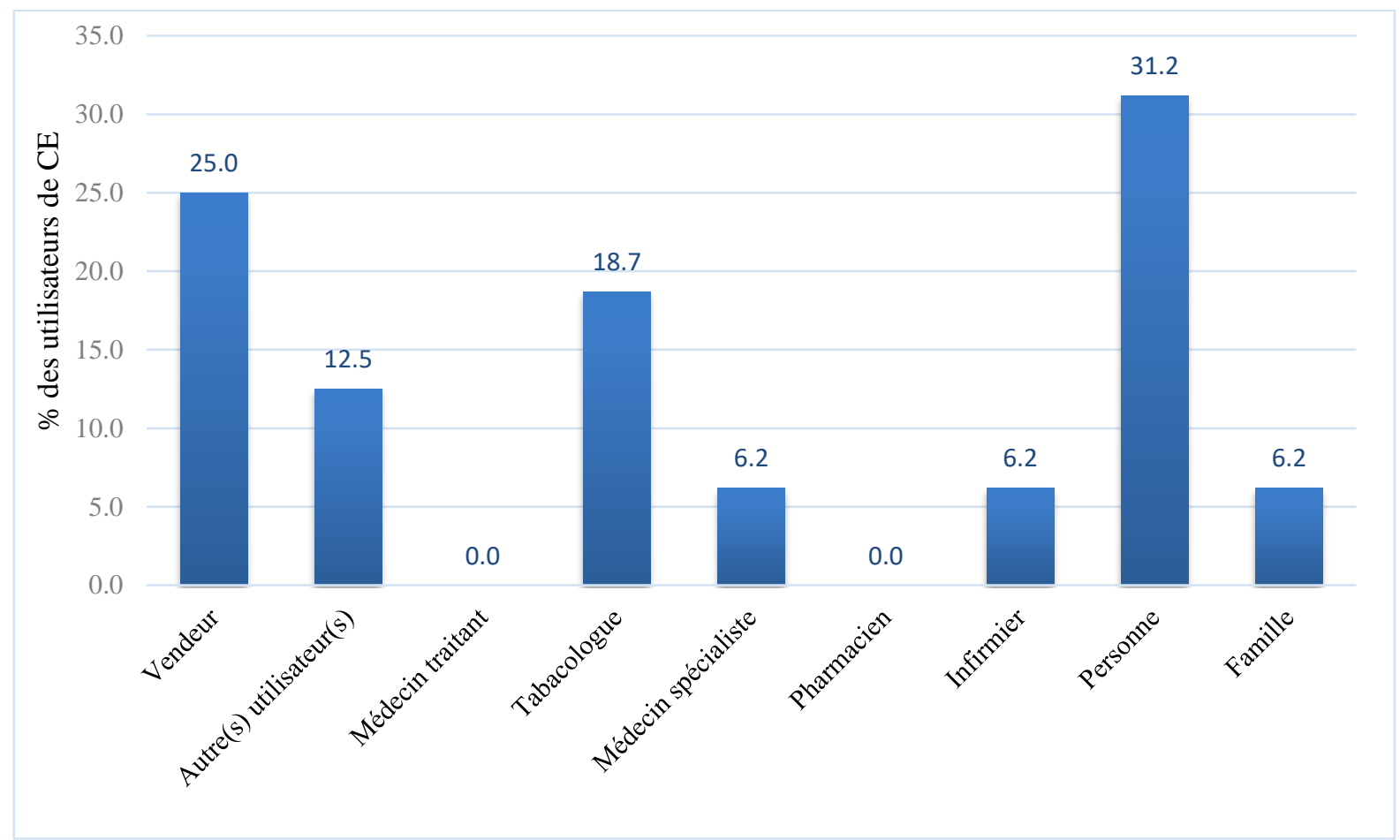

Figure 5 : personne(s) conseillant dans l'utilisation de la CE

\subsection{Rôle du système de santé souhaité par les patients}

16 patients ont répondu à cette partie. $12,5 \%$ des patients auraient aimé que leurs médecins traitants les guident et les suivent dans l'utilisation de la $\mathrm{CE}(\mathrm{n}=2)$ (Figure 6). Aucun n'a répondu vouloir que son médecin traitant l'éduque pour apprendre à gérer sa $\mathrm{CE}$ de lui-même. La moitié des patients souhaitaient que leur médecin traitant ne joue aucun rôle dans l'utilisation de la CE (n=8). 62,5\% ne souhaitaient pas non plus être guidés par un autre professionnel de santé (n=10) (Figure 7). En revanche, sur 15 répondants, 53,3\% des patients pensaient qu'elle devrait être vendue en pharmacie $(n=8)$ car ils estimaient qu'elle est une aide au sevrage $(n=3)$, pour avoir des conseils et des explications (notamment sur les effets indésirables) ( $n=3$ ) ou pour encadrer son utilisation $(n=1)$. Il y avait donc $46,7 \%$ des patients qui ne souhaitaient pas qu'elle soit vendue en pharmacie $(n=7)$ car ils pensaient que cela en limiterait l'utilisation et les possibilités $(n=2)$, en augmenterait son prix $(n=1)$ et qu'elle est un danger pour les adolescents $(n=1)$. 

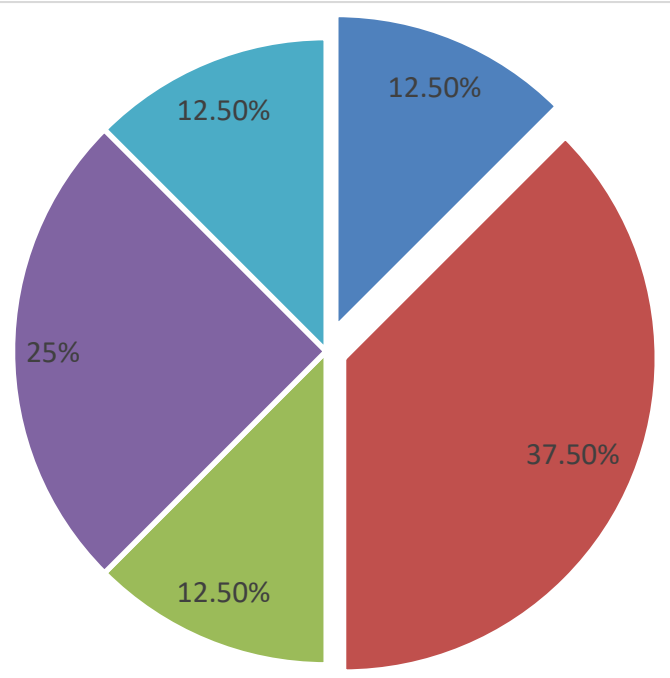

- Etre guidé dans son utilisation, avec un suivi régulier

- Juste des informations (efficacité, effets indésirables...)

- Qu'il me laisse gérer ma consommation et me conseille sur les autres aides

- Aucun, j'aime pouvoir gérer moi-même ma consommation

- Aucun, j'ai déjà toutes les informations dont j'ai besoin

Figure 6 : Rôle du médecin traitant dans l'utilisation de la CE souhaité par les utilisateurs

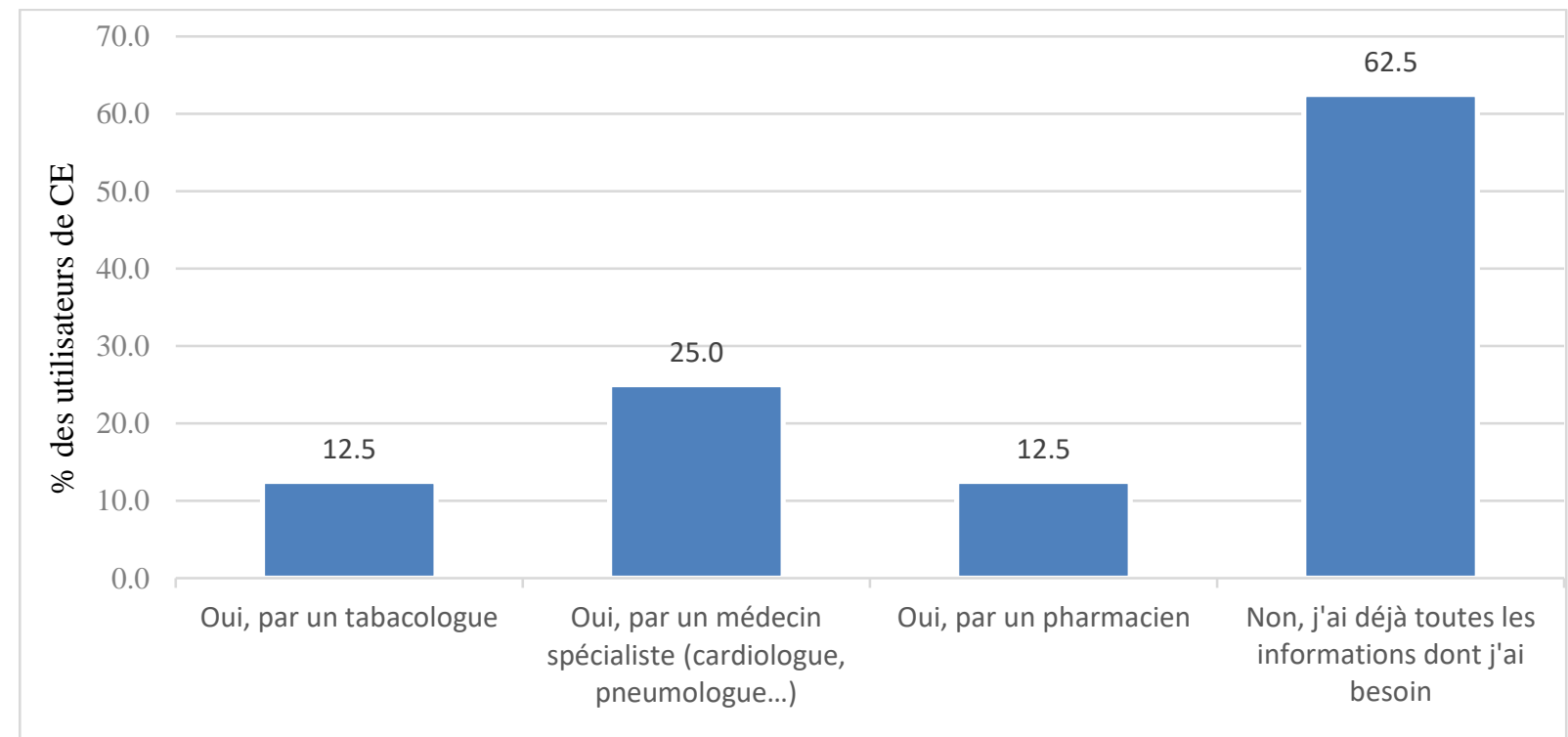

Figure 7 : Souhait des patients d'être guidé par un autre professionnel de santé dans l'utilisation de la $\mathbf{C E}$

\subsection{Les non-utilisateurs de CE}

41 patients ont répondu à la question sur la raison de l'absence d'utilisation de la CE. Plus de la moitié des non-utilisateurs de la CE ne l'ont pas utilisée car ils n'en ont pas eu besoin $(53,7 \%(\mathrm{n}=22)$ ) (Figure 8). Ces patients étaient tous sevrés. 64,3\% des nonexpérimentateurs de CE non sevrés ont exprimé ne pas l'avoir utilisée à cause des incertitudes liées à son utilisation $(\mathrm{n}=9)$. 41,7\% d'entre eux ont cependant répondu qu'ils l'auraient essayée si leur médecin traitant leur avait conseillé. Tous les patients connaissaient la CE (Figure 9). 


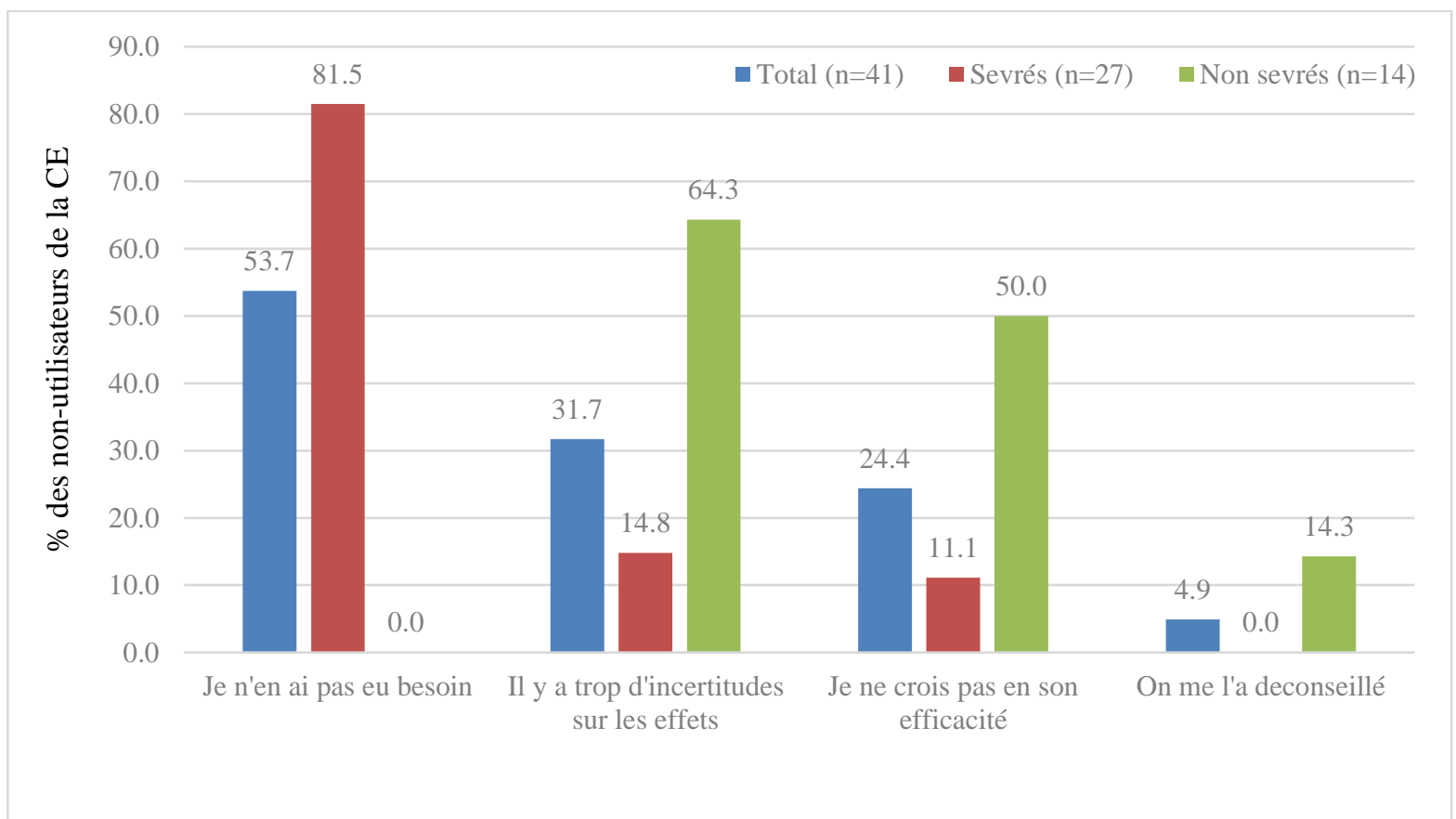

Figure 8 : Raison de la non-utilisation de la CE

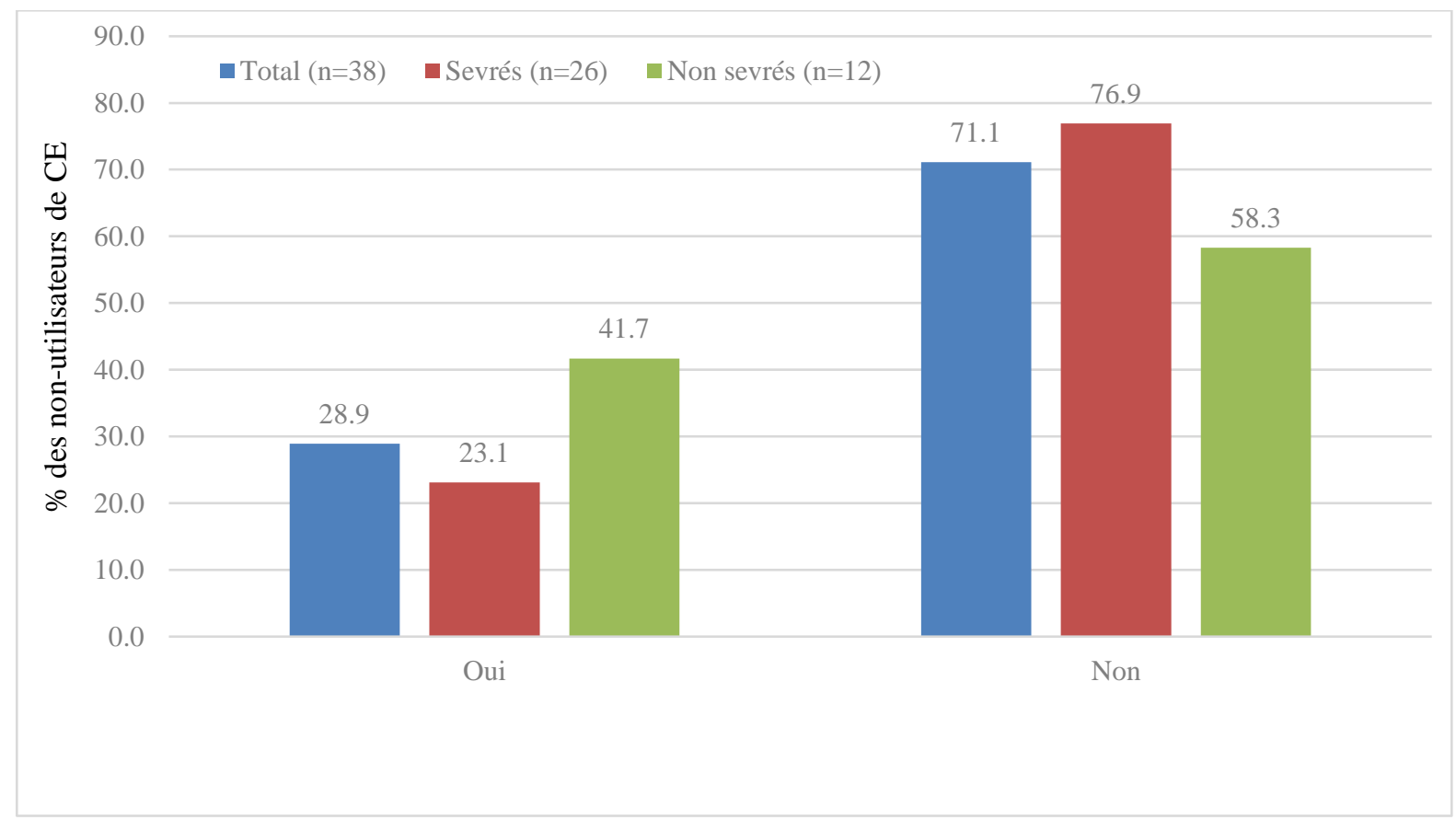

Figure 9 : Utilisation de la CE si le médecin traitant l'avait recommandée

Les patients qui n'auraient pas essayé la CE malgré les conseils de leur médecin traitant $(n=27)$ évoquaient soit, qu'ils n'en ont pas eu besoin $(n=7)$ soit qu'il y avait trop d'incertitudes à son sujet $(n=6)$ ou qu'elle représentait une poursuite d'une intoxication / d'une dépendance $(n=3)$ ou un risque de rechute $(n=1)$. Pour 1 patient, ce n'était pas le rôle du médecin traitant, et pour un autre, elle n'était pas efficace. 


\section{$\underline{\mathrm{IV} / \text { Discussion }}$}

La CE semble être un moyen de plus en plus utilisé dans le sevrage tabagique, y compris chez les patients ayant présenté un SCA. En effet, avant leur SCA, 31,4\% des patients ayant fait une tentative de sevrage l'ont utilisée ainsi que 18,6\% après leur SCA soit $31,4 \%$ des patients n'ayant pas réussi à se sevrer sans aide. Il s'agissait donc du deuxième moyen le plus utilisé après les patchs de nicotine malgré le manque de recommandations de cet outil. Des taux d'utilisation similaires sont aussi retrouvés dans l'étude de Bush AM et al. : 28,6\% de l'ensemble des patients l'avaient utilisée avant leur SCA et 26,5\% 1'avaient utilisée après [46]. Le baromètre santé 2014 montrait que le taux d'expérimentation de la CE suivait un gradient décroissant selon l'âge dans la population générale [28]. Dans notre étude, la $\mathrm{CE}$ a été utilisée par des patients significativement plus jeunes. Il semblerait donc que ce phénomène s'observe aussi chez les patients coronariens.

Le sevrage tabagique était similaire chez les utilisateurs de CE $(63,6 \%)$ et les nonutilisateurs $(66,7 \%)$ après le SCA. La CE n'a donc pas montré d'efficacité sur le sevrage tabagique dans notre échantillon. S'agissant d'une étude observationnelle, ces résultats sont à interpréter avec prudence, les 2 groupes n'étant pas comparables puisque la CE a été utilisée par des patients ayant débuté le tabac plus tôt et avec une consommation tabagique plus élevée avant le SCA. Ceci explique également que seul l'arrêt sans aide a été significativement associé au sevrage, alors que l'efficacité des TNS est démontrée [24]. Concernant la diminution de la consommation, 100\% des utilisateurs de CE après le SCA non sevrés ont diminué leur consommation tabagique $(n=4)$ mais aucune différence $n$ 'a été mise en évidence avec les non-utilisateurs, l'interprétation de ce résultat étant limitée par la faible puissance de notre étude. De plus, notre étude ne prend pas en compte les facteurs confondants tels que l'intensité des symptômes initiaux, la durée d'hospitalisation, les complications au décours, les comorbidités, pouvant influencer la motivation au sevrage. Il existe une perception d'efficacité relative puisque $35,7 \%$ des utilisateurs de CE la citaient comme le moyen le plus efficace soit un taux similaire aux TNS dont les utilisateurs étaient $34,8 \%$ à les considérer comme les moyens les plus efficaces. Seul un quart des utilisateurs de CE l'a arrêtée par manque d'efficacité.

Notre étude est, à notre connaissance, la première à s'intéresser aux vœux de médicalisation du dispositif des patients coronariens et les résultats sont partagés. Elle souligne l'attachement d'une partie des patients à l'autonomie de la CE vis-à-vis du système de santé puisque la moitié des patients souhaitaient que leur médecin traitant ne joue aucun 
rôle dans l'utilisation de la $\mathrm{CE}$ et $62,5 \%$ ne souhaitaient pas être guidé par un autre professionnel de santé. Les professionnels de santé l'ont peu conseillée : l'expérimentation n'a été conseillée par un professionnel de santé que chez 37,5\% des utilisateurs et chez des patients réfractaires au sevrage (80\% d'entre eux étaient non sevrés) ou gros fumeurs (plus d'un paquet de cigarette par jour et fortement dépendant à la nicotine avant le SCA). Seul un quart des patients a eu des conseils dans l'utilisation de la CE par un professionnel de santé. Cette distance, vis-à-vis du système de santé, facilite l'accessibilité du dispositif mais peut en limiter l'efficacité : un fumeur recevant de l'aide par un professionnel de santé et voulant arrêter aura plus de chances d'y parvenir que celui voulant arrêter sans aide [44]. Il existe aussi une tendance à substituer le tabac par la CE puisque $81,8 \%$ des utilisateurs de CE après le SCA étaient toujours consommateurs. L'inscription de la CE dans une stratégie de sevrage suivi par un professionnel de santé pourrait limiter ce phénomène. Une autre partie des patients faisait la demande d'être guidé et suivi par leur médecin traitant dans l'utilisation de la CE (12,5\%) ou de les informer sur l'état actuel des connaissances (37,5\%) et certains auraient aimer être guidé par leur médecin spécialiste, un tabacologue ou un pharmacien (37,5\%). Etant donné l'importance d'améliorer le sevrage tabagique chez ces patients, il apparait important de pouvoir répondre aussi à cette demande. La diffusion de la CE nécessite l'établissement de recommandations claires basées sur des études de qualités afin d'améliorer les connaissances sur la balance bénéfices-risques de l'utilisation de cet outil dans cette population et notamment de la comparer avec les outils actuellement recommandés. Pour faciliter ce type d'étude, la création d'une CE «médicalisée », comme le suggère le HCSP [45], standard avec des constituants de qualité pharmaceutique permettrait de réaliser des comparaisons fiables et dans notre étude, $53,3 \%$ des patients ayant utilisé la CE étaient en faveur de la vente en pharmacie.

La communication prudente autour de la CE semble avoir pour effet néfaste de décourager son utilisation chez les patients non sevrés : dans notre étude, 64,3\% des patients poursuivant une intoxication tabagique et non utilisateurs de CE n'y ont pas eu recours en raison des incertitudes liées à son utilisation bien qu'elle représente un outil de réduction des risques liés au tabagisme y compris sur la fonction myocardique [47]. Cette donnée appuie l'avis du HCSP d'en informer les fumeurs et les professionnels de santé [45]. D'autant plus que $41,7 \%$ de ces patients ont répondu qu'ils l'auraient essayée si leur médecin traitant leur avait conseillé.

Les moyens d'aide au sevrage recommandés par les autorités de santé en prévention secondaire d'un SCA sont principalement les TNS, en cas de dépendance nicotinique, afin 
d'éviter tout symptômes de sevrage et la consultation de tabacologie. Le bupropion et la varenicline peuvent également être utilisés, ainsi que la thérapie cognitivo-comportementale seule ou en association avec un de ces traitements [15]. Dans notre étude, les moyens d'aide au sevrage tabagique recommandés étaient encore peu utilisés après le SCA notamment chez les patients non sevrés qui sont $50 \%$ à ne pas en avoir utilisé $(\mathrm{n}=10)$. La plateforme tabacinfo-service de l'INPES semble encore trop méconnue : aucun patient n'a fait appel à ses services.

Il restait encore 33,9\% des patients de notre échantillon qui n'étaient pas sevrés 1 an après leur SCA. Cette proportion est toutefois plus faible que dans l'étude européenne EUROASPIRE IV où 49,3\% des patients ne sont pas sevrés à 6 mois du SCA. Ce taux de réussite plus élevé peut s'expliquer en partie par un biais dans l'auto déclaration au sevrage qui est parfois surestimée par les patients [48-49]. Il peut exister aussi un biais de sélection : les patients n'ayant pas répondu au questionnaire pouvaient être des patients majoritairement non-sevrés se sentant moins concernés par l'étude.

Notre échantillon était plus jeune que l'ensemble des Français victimes d'un SCA, avec un âge moyen de 53,3 ans contre 65,6 ans dans le registre FAST-MI 2010 où les plus de 75 ans représentent $31,1 \%$ des patients [50]. Ce jeune âge s'explique également par la sélection de patients fumeurs, le tabac étant, avec l'hérédité coronarienne, le principal facteur de risque de SCA du sujet jeune [51]. La proportion de femmes de notre échantillon est similaire à la population générale de ce registre avec respectivement 23,7\% contre 26,9\% [50]. Dans notre échantillon, l'âge moyen des hommes et des femmes était identique. Or, dans la population générale, l'âge moyen des femmes hospitalisées pour un SCA est plus élevé que chez les hommes (74,6 ans pour les femmes contre 64,6 ans pour les hommes) [52]. Une méta-analyse répertoriant les données de plus de 2 millions de patients montre que le risque relatif de maladie coronarienne chez les femmes fumeuses est $25 \%$ plus élevé que chez les fumeurs masculins. La durée du tabagisme a un impact important puisque ce sur risque augmente de $2 \%$ par année de suivi [53]. Le tabac semble donc également impacter l'âge de survenue de la maladie coronarienne chez les femmes. Une des hypothèses pour expliquer ce phénomène est l'association du tabac avec la pilule contraceptive en France dont les risques semblent sous-estimés : 35,5\% des femmes de 20 à 44 ans prenant la pilule contraceptive sont fumeuses et la prévalence du tabagisme chez les femmes de cette tranche d'âge est identique pour celles prenant la pilule comme celles ne la prenant pas [54]. 


\section{$\underline{\text { V/ Conclusion }}$}

Le sevrage tabagique représente un enjeu majeur en prévention secondaire d'un syndrome coronarien aigu pour diminuer la mortalité, les récidives mais aussi la survenue de nombreuses autres maladies secondaires au tabac. La cigarette électronique a fait récemment son apparition comme moyen d'aide au sevrage tabagique et semble être un outil de réduction des risques liés au tabagisme. Elle est, aujourd'hui, utilisée au deuxième rang des moyens d'aide au sevrage derrière les patchs de nicotine chez les patients ayant présenté un syndrome coronarien aigu. Il est donc important d'évaluer l'efficacité de cet outil par des études comparatives de qualité et d'améliorer les connaissances sur les effets indésirables, y compris sur l'utilisation à long terme. 


\section{$\underline{\text { Références bibliographiques }}$}

1. Haute Autorité de Santé [en ligne]. Les syndromes coronaires aigus (SCA). 2007. [consulté le 15 aout 2017]. Disponible sur: https://www.hassante.fr/portail/upload/docs/application/pdf/sca_synthese_biblio.pdf

2. Haute Autorité de Santé [en ligne]. Programme 2007-2010 : Ensemble, améliorons la prise en charge de l'infarctus du myocarde. Bilan d'étape. 2009. [consulté le 15 aout 2017]. Disponible sur: https://www.hassante.fr/portail/upload/docs/application/pdf/2009-04/dossier_presse_idm_2009.pdf

3. Danchin N, Puymirat E, Aissaoui N, Adavane S, Durand E. Epidemiology of acute coronary syndromes in France and in Europe. Ann Cardiol Angeiol (Paris). 2010 Dec ;59 Suppl 2:S37-41.

4. Résultats de la requête: Effectifs de décès [Internet]. Disponible sur http://www.cepidc.inserm.fr/cgi-bin/broker.exe.

5. Haute Autorité de Santé [en ligne]. Prise en charge de l'infarctus du myocarde à la phase aiguë en dehors des services de cardiologie. Conférence de consensus. 23 novembre 2006. [consulté le 15 aout 2017]. Disponible sur: http://sfar.org/wpcontent/uploads/2015/10/2a_HAS_TEXTE-COURT_Prise-en-charge-de$1 \%$ E2\% $80 \% 99$ infarctus-du-myocarde.pdf

6. Haute Autorité de Santé [en ligne]. Indicateurs pour l'amélioration de la qualité et de la sécurité des soins. Prise en charge hospitalière de l'infarctus du myocarde (IDM). Résultats nationaux de la campagne 2015 - Données 2014. Synthèse. 2015. [consulté le 15 aout 2017]. Disponible sur : https://www.hassante.fr/portail/upload/docs/application/pdf/2015-11/synthese_idm_2015_vd.pdf

7. Fox KA, Carruthers KF, Dunbar DR et al. Underestimated and under-recognized: the late consequences of acute coronary syndrome (GRACE UK-Belgian Study). Eur Heart J. 2010.

8. Haute Autorité de Santé [en ligne]. Programme 2007-2010 : Ensemble, améliorons la prise en charge de l'infarctus du myocarde. Bilan 2009. Etape 3 : suivi post infarctus après la sortie de l'hôpital. 2009. [consulté le 15 aout 2017]. Disponible sur: https://www.has-sante.fr/portail/upload/docs/application/pdf/2009-04/etape3_vf.pdf

9. Haute Autorité de Santé [en ligne]. Programme 2007-2010 : Ensemble, améliorons la prise en charge de l'infarctus du myocarde. Bilan 2009. Etape 2 : de la reperfusion à la sortie de l'hôpital. 2009. [consulté le 15 aout 2017]. Disponible sur : https://www.hassante.fr/portail/upload/docs/application/pdf/2009-04/etape2_vf.pdf 
10. World Health Organization [en ligne]. MPOWER : un programme de politiques pour inverser le cours de l'épidémie. 2008. [consulté le 15 aout 2017]. Disponible sur : http://www.who.int/tobacco/mpower/mpower_french.pdf

11. World Health Organization [en ligne]. Tobacco. 2016.[consulté le 15 aout 2017]. Mis à jour en mai 2017. Disponible sur : http://www.who.int/mediacentre/factsheets/fs339/en

12. Ribassin-Majed L, Hill C. Trends in tobacco-attributable mortality in France. Eur J Public Health. 2015 Oct;25(5):824-8

13. Guignard R, Beck F, Richard J-B, Lermenier A, Wilquin J-L, Nguyen-Thanh V. La consommation de tabac en France en 2014 : caractéristiques et évolutions récentes. Évolutions, 2015, $\mathrm{n}^{\circ} 31: 10 \mathrm{p}$

14. ANAES [en ligne]. Méthodes d'évaluation du risque cardio-vasculaire global. Service évaluation en santé publique. 2004. [consulté le 15 aout 2017]. Disponible sur: https://www.hassante.fr/portail/upload/docs/application/pdf/Risque_cardio_vasculaire_rap.pdf

15. Haute Autorité de Santé [en ligne]. Syndromes coronaires aigus et tabac : prise en charge spécifique en cardiologie, au décours d'un syndrome coronarien aigu. 2007. [consulté le 15 aout 2017]. Disponible sur: https://www.hassante.fr/portail/upload/docs/application/pdf/scatabac_synthese_biblio.pdf

16. Organisation Mondiale de la Santé [en ligne]. Genève. Glossaire de la promotion de la santé. 1999. [consulté le 15 aout 2017]. Disponible sur : http://apps.who.int/iris/bitstream/10665/67245/1/WHO_HPR_HEP_98.1_fre.pdf

17. Ministère des Affaires Sociales, de la Santé et des Droits des Femmes [en ligne]. Dossier de presse. Programme national de réduction du tabagisme. 2014. [consulté le 15 aout 2017]; Disponible sur: http://solidarites-sante.gouv.fr/IMG/pdf/250914__Dossier_de_Presse_-_PNRT_2_.pdf

18. Holford TR, Meza R, Warner KE, Meernik C, Jeon J, Moolgavkar SH, et al. Tobacco Control and the Reduction in Smoking-Related Premature Deaths in the United States, 1964-2012. JAMA. 8 janv 2014;311(2): 164.

19. Pirie K, Peto R, Reeves GK, Green J, Beral V, Collaborators MWS, et al. The 21st century hazards of smoking and benefits of stopping: a prospective study of one million women in the UK. The Lancet. 2013;381(9861):133-141.

20. Critchley J, Capewell S. Mortality risk reduction associated with smoking cessation in patients with coronary heart disease: a systematic review. JAMA. 2003:290(1):86-97. 
21. Wilson K, Gibson N, Willan A, Cook D. Effect of smoking cessation on mortality after myocardial infarction: meta-analysis of cohort studies. Arch Intern Med 2000;160(7):939-944.

22. Kotseva K, Wood D, De Bacquer D, De Backer G, Ryden L, Jennings C, et al. EUROASPIRE IV: A European Society of Cardiology survey on the lifestyle, risk factor and therapeutic management of coronary patients from 24 European countries. Eur J Prev Cardiol. Avr 2016;23(6):636-648.

23. Hughes JR, Keely J, Naud S. Shape of the relapse curve and long-term abstinence among untreated smokers. Addiction. 2004;99(1):29-38.

24. Stead LF, Perera R, Bullen C, Mant D, Hartmann-Boyce J, Cahill K, Lancaster T. Nicotine replacement therapy for smoking cessation. Cochrane Database Syst Rev. 2012 Nov 14;11:CD000146.

25. Berlin I. Le tabagisme et la cigarette électronique en France. Presse Med. 2016;45(12):1141-6

26. Office Français de Prévention du Tabagisme. Rapport et avis d'experts sur l'ecigarette. Avec le soutien de la Direction générale de la santé. Mai 2013. 212p.

27. Lermenier-Jeannet A. Tabagisme et arrêt du tabac en 2015. Observatoire français des drogues et des toxicomanies. Fev 2016. 9p.

28. Andler R, Guignard R, Wilquin J-L, Beck F, Nguyen-Thanh V. L'usage de la cigarette électronique en France en 2014. Évolutions, 2015, n³3:6 p

29. Hartmann-Boyce J, McRobbie H, Bullen C, Begh R, Stead LF, Hajek P. Electronic cigarettes for smoking cessation. Cochrane Database Syst Rev. 2016 Sep 14;9:CD010216.

30. McRobbie H, Bullen C, Hartmann-Boyce J, Hajek P. Electronic cigarettes for smoking cessation and reduction. Cochrane Database Syst Rev. 2014;(12):CD010216

31. Rahman MA, Hann N, Wilson A, Mnatzaganian G, Worrall-Carter L. E-Cigarettes and Smoking Cessation: Evidence from a Systematic Review and Meta-Analysis. 2015. PLoS ONE 10(3): e0122544.

32. Kalkhoran S, Glantz SA. E-cigarettes and smoking cessation in real-world and clinical settings: a systematic review and meta-analysis. Lancet Respir Med. Févr 2016 ;4(2) :116-28.

33. Bernstein SL. Electronic cigarettes : more light, less heat needed. Lancet Respir Med. Févr 2016 ;4(2):85-7. 
34. Farsalinos KE, Poulas K, Voudris V, Le Houezec J. Electronic cigarette use in the European Union: analysis of a representative sample of 27460 Europeans from 28 countries. Addiction. 2016;111(11):2032-2040.

35. Etter JF, Bullen C. A longitudinal study of electronic cigarette users. Addict Behav. $2014 \mathrm{Feb} ; 39(2): 491-4$.

36. St.Helen G, Havel C, Dempsey DA, Jacob P, Benowitz NL. Nicotine delivery, retention and pharmacokinetics from various electronic cigarettes : Nicotine retention and PK from e-cigarettes. Addiction. Mars 2016 ;111(3):535-44.

37. Norwegian Institute of Public Health [en ligne]. Health risks associated with the use of electronic cigarettes - Summary in English. 2015. [consulté le 15 aout 2017]. Disponible sur: https://www.fhi.no/globalassets/dokumenterfiler/rapporter/healthrisks-associated-with-e-cigarettes---english-summary-pdf.pdf

38. Thornton JG, Coleman T, Britton J, Cooper S, Watts K, Lewis S, et al. The smoking, nicotine and pregnancy (SNAP) trial: Main results. Archives of Disease in Childhood: Fetal and Neonatal Edition 2011;96:Fa109

39. Allen JG, Flanigan SS, LeBlanc M, Vallarino J, MacNaughton P, Stewart JH, Christiani DC. Flavoring Chemicals in E-Cigarettes: Diacetyl, 2,3-Pentanedione, and Acetoin in a Sample of 51 Products, Including Fruit-, Candy-, and Cocktail-Flavored E-Cigarettes. Environ Health Perspect. 2016 Jun;124(6):733-9.

40. Goniewicz ML, Knysak J, Gawron M, Kosmider L, Sobczak A, Kurek J, et al. Levels of selected carcinogens and toxicants in vapour from electronic cigarettes. Tob Control. Mars 2014 ;23(2) :133-9.

41. Shahab L, Goniewicz ML, Blount BC, Brown J, McNeill A, Alwis KU, et al. Nicotine, Carcinogen, and Toxin Exposure in Long-Term E-Cigarette and Nicotine Replacement Therapy Users: A Cross-sectional Study. Ann Intern Med. 2017;166:390-400.

42. McNeill A, Brose LS, Calder R, Hitchman SC, Hajek P, McRobbie H [en ligne]. Ecigarettes : an evidence update. Public Health Engl. 2015. [consulté le 15 aout 2017]. Disponible sur : https://regulatorwatch.com/wpcontent/uploads/2015/12/Ecigarettes_an_evidence_update_Public_Health_England_FI NAL.pdf

43. Haute Autorité de Santé [en ligne]. AVIS N ${ }^{\circ}$ 2015.0100/AC/SBPP du 4 novembre 2015 du collège de la Haute Autorité de santé sur la nécessité d'actualiser la recommandation de bonne pratique : «Arrêt de la consommation de tabac : du dépistage individuel au maintien de l'abstinence en premier recours » (octobre 2014), 
suite au rapport sur la cigarette électronique du Public Health England. 2015. [consulté le 15 aout 2017]. Disponible sur : https://www.hassante.fr/portail/upload/docs/application/pdf/2015-

11/a_2015_0100_reponse_courrier_dgs_actualisation_rbp_tabac.pdf

44. Haute Autorité de Santé [en ligne]. Recommandation de bonne pratique. Arrêt de la consommation de tabac : du dépistage individuel au maintien de l'abstinence en premier recours. Oct 2014.[Consulté le 15 aout 2017]. Disponible sur: http://www.hassante.fr/portail/upload/docs/application/pdf/2014-

11/reco2clics_arret_de_la_consommation_de_tabac_2014_2014-11-13_10-5148_441.pdf

45. Haut Conseil de la Santé publique (HCSP). Avis relatif aux bénéfices-risques de la cigarette électronique ou e-cigarette étendus en population générale. Revue des Maladies Respiratoires. Fev 2016 ; 33(6) : 509-525.

46. Busch AM, Leavens EL, Wagener TL, Buckley ML, Tooley EM. Prevalence, Reasons for Use, and Risk Perception of Electronic Cigarettes Among Post-Acute Coronary Syndrome Smokers. J Cardiopulm Rehabil Prev. 2016 Sep-Oct;36(5):352-7.

47. Farsalinos KE, Tsiapras D, Kyrzopoulos S, Savvopoulou M, Voudris V. Acute effects of using an electronic nicotine-delivery device (electronic cigarette) on myocardial function: comparison with the effects of regular cigarettes. BMC Cardiovasc Disord. 2014 Jun 23;14:78.

48. Wilcox RG, Hughes J, Roland J. Verification of smoking history in patients after infarction using urinary nicotine and cotinine measurements. Br Med J. 1979 Oct 27;2(6197):1026-8

49. Patrick DL, Cheadle A, Thompson DC, Diehr P, Koepsell T, Kinne S. The validity of self-reported smoking: a review and meta-analysis. Am J Public Health. 1994 Jul;84(7):1086-93

50. Hanssen M, Cottin Y, Khalife K, Hammer L, Goldstein P, Puymirat E, et al. French Registry on Acute ST-elevation and non ST-elevation Myocardial Infarction 2010. FAST-MI 2010. Heart. 2012 May;98(9):699-705

51. Estève JB, Collet JP, Montalescot G. La maladie coronaire du sujet jeune. Sang Thrombose Vaisseaux 2008;20(8):404-9

52. Gabet A, Danchin N, Olié V. Infarctus du myocarde chez la femme : évolutions des taux d'hospitalisation et de mortalité, France, 2002-2013. Bull Epidémiol Hebd. 2016;(7-8):100-8 
53. Huxley RR, Woodward M. Cigarette smoking as a risk factor for coronary heart disease in women compared with men: a systematic review and meta-analysis of prospective cohort studies. Lancet. 2011 Oct 8;378(9799):1297-305

54. Institut National de Prévention et d'Education pour la Santé [en ligne]. Tabac : l'offensive. Dossier de presse. 27 mai 2003. [consulté le 06 aout 2017]. Disponible sur : http://inpes.santepubliquefrance.fr/70000/dp/03/dp030527.pdf 


\section{Annexe 1: Aide à la cotation des actes du service de cardiologie du centre hospitalier de Saint Quentin}

\section{SERVICE DE CARDIOLOGIE SERVICE DE SOINS INTENSIFS CARDIOLOGIQUES}

Centre Hospitalier de Saint-Quentin

\section{COTATIONS ACTES}

Service : SSIC Cardiologie
Date d'entrée:
Date de sortie :
Actes codifiés :

\section{Antécédents}

\begin{tabular}{|c|c|}
\hline 2 & Z96.3 Prösence dfune bioprothedse \\
\hline \multirow[t]{4}{*}{2} & $\begin{array}{l}295.2 \text { Presence dune promise melcanique } \\
\text { cardiague }\end{array}$ \\
\hline & 295.5 Pressence dit atern \\
\hline & 295.1 Pretsence dun PAC \\
\hline & $\begin{array}{l}\text { Z25.0 Prisence dun dispesier dectrenique } \\
\text { cardeque (DEF, DCI. TRC) }\end{array}$ \\
\hline
\end{tabular}

Facteurs de risque cardiovasculaire

\begin{tabular}{|c|c|}
\hline & 140 HTA (primbive) \\
\hline & E78.0 Hyperchalestbrolemia essantielie \\
\hline & E78.2 Hyperilpidemie mixte \\
\hline 2 & $\begin{array}{l}\text { E11.20 DNID insuanorépuerant avec } \\
\text { eompäcations iénales }\end{array}$ \\
\hline 2 & $\begin{array}{l}\text { E11.40 DNID insuincrequitrant avec } \\
\text { complications neuro }\end{array}$ \\
\hline 2 & $\begin{array}{l}\text { E11.30 DNID ineulinerequérart avec } \\
\text { complication cailaire }\end{array}$ \\
\hline & E11.98 DNID sens complication \\
\hline 2 & $\begin{array}{l}\text { E11.90 DNiD insulinorequarart sans } \\
\text { complication }\end{array}$ \\
\hline 2 & E10.8 DiD (type I) avec complications \\
\hline 2 & E10.9 DiD (lype 2) sans complication \\
\hline & E66.03 Surpeids \\
\hline & ES6.90 Cobsite, BMI $30-40$ \\
\hline 2 & E66.91 Cbeside, BMI $40-60$ \\
\hline 2 & E66.92 Cbesats, BMI 50 \\
\hline & F17.241 Tabac \\
\hline & E79.0 Hyperuricèmie asymptomatique \\
\hline
\end{tabular}

\section{Cardiopathie hypertensive}

\begin{tabular}{|c|c|}
\hline 2 & 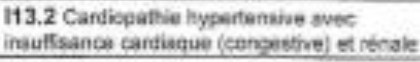 \\
\hline & $\begin{array}{l}\text { 115.9 Hypertensian secondaire, servs } \\
\text { prócision }\end{array}$ \\
\hline & 115.0 Hypertansian vasculo-rknale \\
\hline & $\begin{array}{l}115.2 \text { Hypertensian secondaire a des } \\
\text { atteintes endocriniernes }\end{array}$ \\
\hline
\end{tabular}

\section{Mvocardiopathie}

\begin{tabular}{|c|c|}
\hline 2 & I50.1 insumbance cardiaque conoestive \\
\hline & $42.0 \mathrm{CMO}$ \\
\hline 3 & R57.0 Choc cardegenique \\
\hline 2 & Ata Alscile \\
\hline & 140.0 Byocardte agut infectituse \\
\hline 2 & 142.0 Myocarticpathin alcoekque \\
\hline
\end{tabular}

\section{Cardiopathie ischómique}

\begin{tabular}{|c|c|}
\hline \multirow[t]{3}{*}{2} & 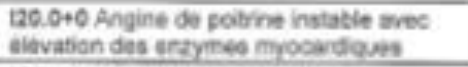 \\
\hline & 120.8 Angine de peitrire foffert \\
\hline & 125.1 Caralopathie athatoeclitrouse \\
\hline 3 & 121.00 IDM antetileur \\
\hline 3 & $\begin{array}{l}\text { 121. oco IDAt antirieur de } 24 \text { teures ou } \\
\text { eoins }\end{array}$ \\
\hline 3 & $\begin{array}{l}\text { i21.100 ich interour de } 24 \text { haves oe } \\
\text { moins }\end{array}$ \\
\hline 3 & 121.16 OM intartaur \\
\hline 3 & 121.20 IOM autres focalisabions \\
\hline 3 & $\begin{array}{l}\text { 12t. } 300 \text { idut localsastion non cribitede de } 24 \\
\text { heures ou mons }\end{array}$ \\
\hline 2. & 121.40 IDAt scus andocandipue \\
\hline \multirow[t]{3}{*}{3} & $\begin{array}{l}121.400 \text { IOM sous-endocandique de } 24 \\
\text { heures ou meins }\end{array}$ \\
\hline & 120.1 Angor spastique vitritio \\
\hline & 125.2 infarchus du myocande, encien \\
\hline
\end{tabular}

\section{Complications infarctus}

\begin{tabular}{|c|c|}
\hline 2 & 1230 Hêmopericarde poot lou \\
\hline 2 & 123.1 CLA post IOM \\
\hline 2 & 123.2 CW post IDM! \\
\hline 2 & $\begin{array}{l}\text { 123.J Rupture de la paroi cardiaque sans } \\
\text { hemopericarde post iOM }\end{array}$ \\
\hline 2 & 123.4 Rupture des cortages post IDU \\
\hline 2 & $\begin{array}{l}123.6 \text { Thrombicse de fortilletle, be favicule } \\
\text { et du ventricule post iDM }\end{array}$ \\
\hline
\end{tabular}




\section{Annexe 2: Questionnaire}

Bonjour,

Je vous adresse ce courrier car j'effectue un travail de thèse avec le Dr Chevalier (cardiologue au CH de Saint Quentin), concernant le tabac et le sevrage tabagique après un évènement coronarien aigu.

Pour cela, je vous serais reconnaissant de bien vouloir remplir ce questionnaire qui ne vous prendra que 5 minutes.

Une enveloppe de retour vous est jointe dans le courrier.

Si vous souhaitez obtenir les résultats de cette thèse, vous pouvez laisser votre adresse mail à la fin du questionnaire.

En vous remerciant par avance de votre participation et de la grande aide que vous m'apporterez dans cette thèse.

Cordialement,

Yann Bellenguez (interne de médecine générale)

\section{$\underline{\text { A/ Renseignements }}$}

1/ Quel est votre âge ?

2/ Sexe : -Féminin -Masculin

3/ Situation professionnelle :

-cadre supérieur

-salarié

-Retraité

-Sans activité

-En invalidité / Allocation Adulte Handicapé -profession libérale

-fonctionnaire

-Chômage

-En formation / Etudiant

\section{4/ Quel est votre niveau d'étude ?}
-Sans diplôme
-Collège / CAP / BEP
-Niveau secondaire, lycée
-Baccalauréat
$-\mathrm{Bac}+2$
-Au-delà de Bac +2 


\section{B/ Histoire du tabagisme avant l'infarctus}

5/ A quel âge avez-vous commencé à fumer régulièrement du tabac ?

6/ Avant votre infarctus, que fumiez-vous principalement ?

$\begin{array}{ll}\text {-Cigarettes en paquet / Tubes } & \text {-Cigarettes roulées } \\ \text {-Cigarillos } & \text {-Cigares } \\ \text {-Pipes } & \text {-Joints }\end{array}$

7/ Avant votre infarctus, combien de fois par jour fumiez-vous du tabac en moyenne?

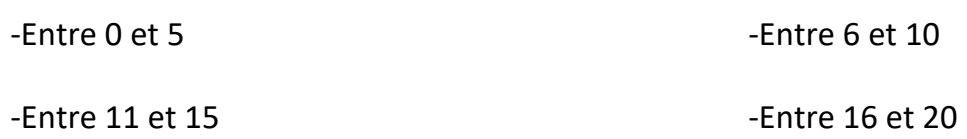

8/ Avant votre infarctus, combien de temps, en moyenne, après votre réveil fumiez-vous du tabac ?
-Dans les 5 minutes
-De 6 à 30 minutes
-De 31 à 60 minutes
-Plus de 60 minutes

9/ Avant votre infarctus, aviez-vous déjà effectué des tentatives d'arrêt du tabac ( 0 cigarettes pendant au moins 7 jours) ?

-Oui -Non

Si oui, combien de tentatives ?.

10/ Avant votre infarctus, quelle était votre motivation à arrêter de fumer ?

Pas du tout motivé $\begin{array}{llllllllllll}0 & 1 & 2 & 3 & 4 & 5 & 6 & 7 & 8 & 9 & 10 & \text { Extrêmement motivé }\end{array}$ 
11/ Avant votre infarctus, quels moyens d'aide à l'arrêt du tabac aviez-vous déjà utilisé ? (Plusieurs choix possibles pour chaque tentative)

-Patchs de nicotine.

-Autres substituts nicotiniques (gommes, comprimés, spray, inhaleur)

-Cigarette électronique contenant de la nicotine.

-Cigarette électronique sans nicotine.

-Consultation avec un tabacologue

-Acupuncture

-Hypnose

-Homéopathie

-Champix (Varenicline)

-Zyban (Bupropion)

-Psychothérapie de soutien / cognitivo-comportementales.....

-Arrêt sans aide (« volonté seule »)

-Internet / Telephone / Applications... (Tabac Info Service...)....

-Aucun, je n'avais jamais essayé d'arrêter de fumer

-Autre (veuillez préciser) :

\begin{tabular}{|c|c|c|c|c|c|}
\hline \begin{tabular}{|c|} 
1ere \\
tentative
\end{tabular} & $2 e$ & $3 e$ & $4 \mathrm{e}$ & $5 e$ & Ensuite \\
\hline & & & & & \\
\hline & & & & & \\
\hline & & & & & \\
\hline & & & & & \\
\hline & & & & & \\
\hline & & & & & \\
\hline & & & & & \\
\hline & & & & & \\
\hline & & & & & \\
\hline & & & & & \\
\hline & & & & & \\
\hline & & & & & \\
\hline & & & & & \\
\hline & & & & & \\
\hline & & & & & \\
\hline
\end{tabular}

\section{C/ Depuis votre infarctus}

12/ Après votre infarctus, quelle était votre motivation à arrêter de fumer ?

Pas du tout motivé $\quad \begin{array}{llllllllllll}0 & 1 & 2 & 3 & 4 & 5 & 6 & 7 & 8 & 9 & 10 & \text { Extrêmement motivé }\end{array}$

13/ Actuellement, fumez-vous toujours du tabac?

-Oui, occasionnellement Passez à la question 16

-Oui, tous les jours Passez à la question 16

-Non 
14/ Si vous avez arrêté de fumer, depuis combien de temps avez-vous arrêté ?

15/ Quel est votre degré de confiance à ne pas reprendre le tabac ?

Pas du tout confiant $\begin{array}{llllllllllll}0 & 1 & 2 & 3 & 4 & 5 & 6 & 7 & 8 & 9 & 10 & \text { Je suis sûr de ne pas reprendre }\end{array}$

------> Passez à la question 18

16/ Si vous continuez de fumer, combien de fois par jour fumez-vous du tabac en moyenne?

$\begin{array}{ll}\text {-Entre } 0 \text { et } 5 & \text {-Entre } 6 \text { et } 10 \\ \text {-Entre } 11 \text { et } 15 & \text {-Entre } 16 \text { et } 20 \\ \text {-Entre } 21 \text { et } 30 & \text {-Plus de } 30\end{array}$

$17 /$ Si vous continuez de fumer, combien de temps après votre réveil fumez-vous du tabac ?

-Dans les 5 minutes -Entre 6 et 30 minutes

-Entre 31 et 60 minutes $\quad$-Plus de 60 minutes

Utilisez-vous un substitut nicotinique (gomme, comprimé, inhaleur, spray, patch, cigarette électronique contenant de la nicotine) avant votre $1^{\text {ère }}$ cigarette ?

-Oui -Non

18/ Depuis votre infarctus, combien de tentatives d'arrêt ( 0 cigarettes pendant au moins de 7 jours) avezvous effectué ? 
19/ Depuis votre infarctus, quels moyens d'aide à l'arrêt du tabac avez-vous utilisé ? (Plusieurs choix possibles pour chaque tentative)

-Patchs de nicotine.

-Autres substituts nicotiniques (patchs, gommes, comprimés, spray, inhaleur)

-Cigarette électronique contenant de la nicotine.

-Cigarette électronique sans nicotine.

-Consultation avec un tabacologue

-Acupuncture

-Hypnose

-Homéopathie

-Champix (Varenicline)

-Zyban (Bupropion)

-Psychothérapie de soutien / cognitivo-comportementales

-Arrêt sans aide (« volonté seule »)

-Internet / Telephone / Applications... (Tabac Info Service...)......

-Aucun, je n'avais jamais essayé d'arrêter de fumer

-Autre (veuillez préciser) :

\begin{tabular}{|c|c|c|c|c|c|}
\hline $\begin{array}{c}\text { 1ere } \\
\text { tentative }\end{array}$ & $2 e$ & $3 e$ & $4 e$ & $5 e$ & Ensuite \\
\hline & & & & & \\
\hline & & & & & \\
\hline & & & & & \\
\hline & & & & & \\
\hline & & & & & \\
\hline & & & & & \\
\hline & & & & & \\
\hline & & & & & \\
\hline & & & & & \\
\hline & & & & & \\
\hline & & & & & \\
\hline & & & & & \\
\hline & & & & & \\
\hline & & & & & \\
\hline & & & & & \\
\hline
\end{tabular}

20/ Quel(s) moyen(s) jugez vous le(s) plus efficace(s) ? 


\section{D/ Parmi les personnes ayant utilisé la cigarette électronique :}

21/ Qui vous a motivé / conseillé la cigarette électronique ? (Plusieurs choix possible)

$\begin{array}{ll}\text {-Famille } & \text {-Amis } \\ \text {-Publicité } & \text {-Médecin traitant }\end{array}$

-Autre professionnel de santé (médecin spécialiste, pharmacien, infirmier...)

-Autre :

22/ Etes-vous toujours utilisateur de cigarette électronique?
-Oui, quotidiennement
-Oui, occasionnellement
-Non je n'en ai plus besoin
-Non, ça ne m’a pas suffisamment aidé

23/ Qui vous conseille ou vous a conseillé dans son utilisation ? (Plusieurs choix possibles)
-Un vendeur
-Un ou des autre(s) utilisateur(s)
-Votre médecin traitant
-Un tabacologue
-Un médecin spécialiste (cardiologue, pneumologue...)
-Un pharmacien
-Un infirmier
-Personne
-Autre :

24/ Quel rôle aimeriez-vous que votre médecin traitant occupe concernant la cigarette électronique ? -Juste des informations (efficacité, les effets secondaires...)

-Quelques conseils pour apprendre à gérer moi-même ma consommation de cigarette électronique -Etre guidé sur son utilisation, avec un suivi régulier

-Qu'il me laisse gérer ma cigarette électronique et qu'il me conseille sur les autres aides disponibles -Aucun, j'aime pouvoir gérer moi-même ma consommation -Aucun, j’ai déjà tous les conseils / toutes les informations dont j'ai besoin 
25/ Souhaiteriez-vous être guidé dans son utilisation par un autre professionnel de santé ? (Plusieurs réponses possibles)
-Oui, par un tabacologue
-Oui, par un médecin spécialiste (cardiologue, pneumologue...)
-Oui, par un pharmacien
-Oui, par un infirmier

-Autre :

-Non, j'ai déjà toutes informations dont j'ai besoin

26/ Pensez-vous que la cigarette électronique devrait être vendue en pharmacie ?

-Oui -Non

Pourquoi ?

\section{E/ Parmi les personnes n'ayant pas utilisé la cigarette électronique}

27/ Pourquoi ne l'avez-vous pas essayée ? (Plusieurs choix possibles)

-Je n'en ai pas eu besoin

-Je ne crois pas en son efficacité

-On me l'a déconseillée

$\rightarrow$ Qui ?
-Il y a encore trop d'incertitudes sur les effets

-Je ne connaissais pas la cigarette électronique

28/ Auriez-vous essayé la cigarette électronique si votre médecin traitant vous l’avait conseillée ?

-Oui

-Non

Pourquoi ? 


\section{$\underline{\text { Annexe } 3 \text { : Test de Fagerström simplifié }}$}

1. Combien de cigarettes fumez-vous par jour ?

10 ou moins...................... 0

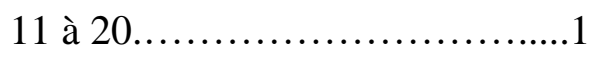

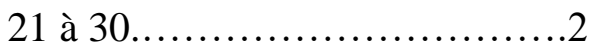

31 ou plus.......................... 3

2. Dans quel délai après le réveil fumez-vous votre première cigarette ?

Moins de 5 minutes................ 3

6 à 30 minutes...................2

31 à 60 minutes...................1

Après plus d' 1 heure..............0

Interprétation selon les auteurs :

- 0-1 : pas de dépendance ;

-2-3 : dépendance modérée ;

- 4-5-6 : dépendance forte. 
Sevrage tabagique en prévention secondaire d'un syndrome coronarien aigu : la place de la cigarette électronique

\section{Résumé :}

Introduction : Le sevrage tabagique est un enjeu majeur en prévention secondaire d'un syndrome coronarien aigu (SCA). La cigarette électronique (CE) a fait récemment son apparition parmi les moyens d'aide au sevrage. Nous avons étudié l'utilisation, l'efficacité et les souhaits de «médicalisation » de la CE dans ce contexte.

Méthodes : Etude épidémiologique prospective avec inclusion des patients adultes fumeurs hospitalisés du $1^{\text {er }}$ juin 2015 au 31 mars 2016 au Centre Hospitalier de Saint Quentin (02) pour un SCA (sauf angor instable), à l'exclusion des patients de plus de 75 ans et/ou avec antécédent personnel de SCA, questionnés à 1 an de leur SCA.

Résultats : 81 patients ont été inclus. 59 questionnaires (73\%) ont été renvoyés et analysés. L'âge moyen était de 53,3 ans. Il y avait 45 hommes (76,3\%) et 14 femmes (23,7\%). 11 patients (18,6\%) avaient utilisé au moins une fois la CE avant le SCA et également $11(18,6 \%)$ après. C'était le deuxième moyen de sevrage le plus utilisé après les patchs de nicotine. A un an du SCA, 39 patients $(66,1 \%)$ étaient sevrés : 63,6\% (7/11) des utilisateurs de CE après le SCA contre 66,7\% (32/48) des non-utilisateurs $(p=0,848)$. Après analyse multivariée, seul l'arrêt sans aide était associé au sevrage $(p=0,013)$. Les utilisateurs de CE étaient significativement plus jeunes, fumaient plus avant le SCA et avaient débuté le tabac plus tôt. 50\% des utilisateurs de CE auraient souhaité être informés par leur médecin traitant et 53,3\% qu'elle soit vendue en pharmacie.

Conclusion : La CE est le deuxième moyen de sevrage le plus utilisé après un SCA. Des études de qualité sont indispensables pour mieux connaître son efficacité et sa sécurité.

Mots clés : cigarettes électroniques, sevrage tabagique, syndrome coronarien aigu, prévention secondaire, épidémiologie

Smoking cessation in secondary prevention of acute coronary syndrome: the role of the electronic cigarette

\section{$\underline{\text { Abstract: }}$}

Introduction: Smoking cessation is a major issue in secondary prevention of acute coronary syndrome (ACS). The electronic cigarette (EC) has recently come up as a means to help with ceasing. We have studied the use, efficiency and wishes for a "medicalization" of the EC in this context. Methods: Prospective epidemiological study with inclusion of adult smoker patients hospitalized from 1 June 2015 to 31 March 2016 at the St Quentin Hospital (Picardy, France) for an ACS (except unstable angina), excluding patients over 75 years and/or with personal history of ACS, interviewed 1 year after their ACS.

Results: 81 patients were included. 59 questionnaires (73\%) were returned and analyzed. The average age was 53.3 years. There were 45 men $(76.3 \%)$ and 14 women $(23.7 \%) .11$ patients $(18.6 \%)$ had used the EC at least once before their ACS and $11(18.6 \%)$ had used it after. That was the second most used smoking cessation method after the nicotine patches. One year after their SCA, 39 patients $(66.1 \%)$ had stopped smoking: 63.6\% (7/11) of EC users after the ACS against 66.7\% (32/48) of non-users $(p=0.848)$. After multivariate analysis, only cessation without help was associated with ceasing $(p=0.013)$. CE users were significantly younger, were smoking more before their ACS, and had started smoking earlier. $50 \%$ of CE users would have appreciated to be better informed by their general practitioner and $53.3 \%$ expressed themselves in favor of a sale in pharmacies.

Conclusion: EC is the second most common means of smoking cessation after an ACS. Further comparative studies are essential to know more about its efficiency and safety.

Key Words: Electronic Cigarettes, smoking cessation, acute coronary syndrome, secondary prevention, epidemiology 